\title{
A Feynman-Kac-type formula for Lévy processes with discontinuous killing rates
}

\author{
Kathrin Glau 1
}

Received: 16 February 2015 / Accepted: 26 January 2016 / Published online: 31 May 2016

(c) The Author(s) 2016. This article is published with open access at Springerlink.com

\begin{abstract}
The challenge to fruitfully merge state-of-the-art techniques from mathematical finance and numerical analysis has inspired researchers to develop fast deterministic option pricing methods. As a result, highly efficient algorithms to compute option prices in Lévy models by solving partial integro-differential equations have been developed. In order to provide a solid mathematical foundation for these methods, we derive a Feynman-Kac representation of variational solutions to partial integro-differential equations that characterize conditional expectations of functionals of killed time-inhomogeneous Lévy processes. We allow a wide range of underlying stochastic processes, comprising processes with Brownian part as well as a broad class of pure jump processes such as generalized hyperbolic, multivariate normal inverse Gaussian, tempered stable, and $\alpha$-semistable Lévy processes. By virtue of our mild regularity assumptions as to the killing rate and the initial condition of the partial integro-differential equation, our results provide a rigorous basis for numerous applications in financial mathematics and in probability theory. We implement a Galerkin scheme to solve the corresponding pricing equation numerically and illustrate the effect of a killing rate.
\end{abstract}

Keywords Time-inhomogeneous Lévy process $\cdot$ Killing rate $\cdot$ Feynman-Kac representation - Weak solution, variational solution - Parabolic evolution equation · Partial integro-differential equation · Pseudo-differential equation · Nonlocal operator · Fractional Laplace operator · Sobolev-Slobodeckii spaces · Option pricing - Laplace transform of occupation time - Employee option · Galerkin method

Mathematics Subject Classification (2010) 60G51 - 65M06 · 35S10 • 47G20 • 47G30

JEL Classification C10 - C63

$\bowtie$ K. Glau

kathrin.glau@tum.de

1 Center for Mathematics, Technical University of Munich, Munich, Germany 


\section{Introduction}

Feynman-Kac formulas play a distinguished role in probability theory and functional analysis. Ever since their birth in 1949, Feynman-Kac-type formulas have been a constant source of fascinating insights in a wide range of disciplines. They originate in the description of particle diffusion by connecting Schrödinger's equation and the heat equation to the Brownian motion; see [31]. A type of Feynman-Kac formula also figures at the beginning of modern mathematical finance: In their seminal article of 1973 [6], Black and Scholes wrote their Nobel Prize-winning option pricing formula by expressing the price as a solution to a partial differential equation, thereby rediscovering Feynman and Kac's deep link.

The fundamental contribution of Feynman-Kac formulas is to link stochastic processes to solutions of deterministic partial differential equations. Thus they also establish a connection between probability theory and numerical analysis, two disciplines that have evolved largely separately. Although both enjoy great success, transfer between them has remained only incidental. This may very well be the reason for applications of Feynman-Kac still appearing so surprisingly fresh. In computational finance, they enable the development of option pricing methods by solving deterministic evolution equations. These have proved to be highly efficient, particularly when compared to Monte Carlo simulation. Thus, like other deterministic methods, they come into play whenever efficiency is essential and the complexity of the pricing problem is not too high. This is the case for recurring tasks, such as calibration and real-time pricing, and over the last few decades has given rise to extensive research in computing option prices by solving partial differential equations. The challenge to extend these methods to price options in advanced jump models has furthermore inspired researchers in recent years to develop highly efficient and widely applicable algorithms; see for instance [11, 22, 23, 41, 25].

In this article, we derive a Feynman-Kac-type formula so as to provide a solid mathematical basis for fast option pricing in time-inhomogeneous Lévy models using partial integro-differential equations (PIDEs). While large parts of the literature focus on numerical aspects of these pricing methods, only little is known about the precise link between the related deterministic equations and the corresponding conditional expectations representing option prices. Under which conditions is there a Feynman-Kac-type formula linking option prices given by conditional expectations with solutions to evolution equations?

In order to further specify the problem, we focus on time-inhomogeneous Lévy models and options whose path-dependence may be expressed by a killing rate. In this setting with $\mathcal{A}=\left(\mathcal{A}_{t}\right)_{t \in[0, T]}$, the Kolmogorov operator of a time-inhomogeneous Lévy process, a killing rate (or potential) $\kappa:[0, T] \times \mathbb{R}^{d} \rightarrow \mathbb{R}$, for which we write $\kappa_{t}(x):=\kappa(t, x)$, a source $f:[0, T] \times \mathbb{R}^{d} \rightarrow \mathbb{R}$ and an initial condition $g: \mathbb{R}^{d} \rightarrow \mathbb{R}$, the Kolmogorov equation is of the form

$$
\begin{aligned}
\partial_{t} u+\mathcal{A}_{T-t} u+\kappa_{T-t} u & =f, \\
u(0) & =g .
\end{aligned}
$$

Adopting a heuristic approach, one would typically assume that (1.1) has a classical solution $u$. If this solution is sufficiently regular to allow an application of Itô's 
formula and moreover satisfies an appropriate integrability condition, the FeynmanKac-type representation

$$
\begin{aligned}
& u\left(T-t, L_{t}\right) \\
& =E\left[g\left(L_{T}\right) \mathrm{e}^{-\int_{t}^{T} \kappa_{h}\left(L_{h}\right) \mathrm{d} h}+\int_{t}^{T} f\left(T-s, L_{s}\right) \mathrm{e}^{-\int_{t}^{s} \kappa_{h}\left(L_{h}\right) \mathrm{d} h} \mathrm{~d} s \mid \mathcal{F}_{t}\right]
\end{aligned}
$$

follows by standard arguments and taking conditional expectations; see (8.6) and (8.7) below for a detailed derivation. Then, the conditional expectation (1.2) can be obtained by solving the Kolmogorov equation (1.1) by means of a deterministic numerical scheme. Such an argumentation hinges on a strong regularity assumption on the solution $u$ and thus implicitly on the data $g, f, \mathcal{A}$ and $\kappa$ of the equation. This constitutes a serious restriction on the applicability of such a heuristic approach.

To do justice to the complexities of financial applications, we pay special attention to identifying appropriate conditions for the validity of (1.2) for financial applications. Often, discontinuous killing rates constitute a natural choice, as we show in several detailed examples in Sect. 5. In particular, indicator functions as killing rates turn out to be key to a wide variety of applications, both in mathematical finance and in probability theory. As one typical application, we propose and study a flexible family of employee options in Sect. 5.1 and illustrate the numerical effect of such killing rates in Sect. 6. The fundamental role of killing rates of indicator-type is killing the process outside a specified domain, which makes them attractive for applications. Moreover, they are closely related to occupation times and exit times of stochastic processes, as we outline in Sects. 5.3 and 5.4. We furthermore find that discontinuous killing rates form a common root of exit probabilities of stochastic processes and the distribution of supremum processes. As such, they apply to the prices of path-dependent options like those of barrier, lookback and American type. In view of these considerations, which are both of a theoretical and applied nature, we also want to allow non-smooth and even discontinuous killing rates in the Kolmogorov equation (1.1).

Discontinuities in the killing rate $\kappa$ result in non-smoothness of the solution $u$ of (1.1). In particular, one cannot expect $u \in C^{1,2}$ : If $u(0) \neq 0$ and $\kappa_{t}=\kappa:=\mathbb{1}_{(-\infty, 0)^{d}}$ for every $t \in[0, T]$ in (1.1), then $x \mapsto u(t, x) \in C^{2}$ implies $x \mapsto \kappa(x) u(t, x) \in C$, which obviously is a contradiction. Hence, for our purposes, the assumption that Itô's formula can be applied to the solution $u$ is futile. Neither is it reasonable to assume that (1.1) has a classical solution. Let us emphasize that such irregularity is not only inherent in (1.1) if the killing rate is discontinuous, but also a typical feature of Kolmogorov equations for other path-dependent options. Prominent examples are boundary value problems related to barrier options in Lévy models as well as free boundary value problems for American options. In each of these cases, the use of a generalized solution concept is called for.

Among the possible generalizations of classical solutions of partial differential equations, we find that viscosity and weak solutions are the ones that are most commonly discussed. Viscosity solutions directly abstract from pointwise solutions by introducing comparison functions that are sufficiently regular, while the root of weak solutions is the problem formulation in a Hilbert space. Conceptually, both have their 
advantages. From a numerical perspective, viscosity solutions relate to finite difference schemes, whereas weak solutions are the theoretical foundation of Galerkin methods, a rich class of versatile numerical methods to solve partial differential equations. Relying on their elegant Hilbert space formulation, Galerkin methods by their very construction lead to convergent schemes as well as to a lucid error analysis. They furthermore distinguish themselves by their enormous flexibility towards problem types as well as compression techniques. Both theory and implementation of Galerkin methods have experienced a tremendous advancement over the past 50 years. They have become indispensable for today's technological developments in such diverse areas as aeronautical, biomechanical and automotive engineering.

In mathematical finance, Galerkin pricing algorithms have been developed for various applications, even for basket options in jump models. Furthermore, numerical experiments and error estimates have confirmed their efficiency both in theory as well as in practice; see for instance [33, 37, 34, 23]. We present the implementation of a related Galerkin method to price call options adjusted with a killing rate in Sect. 6. Furthermore, Galerkin-based model reduction techniques have a great potential in financial applications; see [12, 38, 19, 40, 20].

Feynman-Kac representations for viscosity solutions with application to option pricing in Lévy models have been derived in [10] and [11]. Results linking jump processes with Brownian part to variational solutions had already been proved earlier in [5]. However, in order to cover some of the most relevant financial models, we have to consider pure jump processes, i.e., processes without a Brownian component, as well. Pure jump Lévy models have been shown to fit market data with high accuracy and have enjoyed considerable popularity; see for instance [13, 43, 9]. Moreover, statistical analysis of high-frequency data supports the choice of pure jump models; see [1].

We realize that pure jump processes differ significantly from processes with a Brownian part. The Brownian component translates to a second order derivative in the Kolmogorov operator, while the pure jump part corresponds to an integro-differential operator of a lower order of differentiation. Accordingly, the second order derivative is only present in Kolmogorov operators of processes with a Brownian component. As a consequence, the solution to the Kolmogorov equation of a pure jump Lévy process does not lie in the Sobolev space $H^{1}$, the space of square-integrable functions with a square-integrable weak derivative. Therefore, we need a more general solution space. In order to make an appropriate choice, recall that Lévy processes are nicely characterized through the Lévy-Khinchine formula by the Fourier transform of their distribution or, equivalently, by the symbol, which is the Fourier representation of the operator. Moreover, the symbol is typically available in terms of an explicit parametric function and as such is the key quantity to parametric Lévy models. For a wide range of processes, the asymptotic behavior of the symbol ensures that the solution of the Kolmogorov equation belongs to a Sobolev-Slobodeckii space, i.e., it has a derivative of fractional order. Even more, parabolicity with respect to SobolevSlobodeckii spaces of the Kolmogorov equations related to Lévy processes has been characterized in terms of growth conditions on the symbol in [18].

To allow typical initial conditions, such as the payout function of a call option in logarithmic variables and the Heaviside step function that relates to distribution functions, we base our analysis more generally on exponentially weighted 
Sobolev-Slobodeckii spaces. We therefore generalize the characterization of parabolicity to time-inhomogeneous Lévy processes and to exponentially weighted SobolevSlobodeckii spaces. In [14], existence and uniqueness of weak solutions in exponentially weighted Sobolev-Slobodeckii spaces for Kolmogorov equations related to time-inhomogeneous Lévy processes and a Feynman-Kac formula have been established. Here, we generalize these results to solutions of Kolmogorov equations related to time-inhomogeneous Lévy processes with possibly discontinuous killing rates. Technically, the present setting is more difficult since the Fourier transform of the solution is not explicitly available, and moreover, the solution is not sufficiently regular for an application of Itô's formula.

The fruitful relation between pseudo-differential operators (PDOs) and Markov processes via their symbols has already been extensively used to establish existence of stochastic processes; see for instance the monographs of Jacob [26, 27, 28]. For a short overview on the different approaches to construct Feller processes and the use of pseudo-differential calculus in this context, see Chapter III in [7]. Let us observe that our question is of a different nature. In our case, existence of the stochastic process $L$ is guaranteed, and we establish a Feynman-Kac-type representation of the form (1.2) on this basis. An interesting feature of our approach is that we do need not impose growth conditions on the (higher-order) derivatives of the symbol as in the standard symbolic calculus. Our approach is more closely related to [24], where a class of martingale problems is solved by tracing back the existence of the processes to parabolicity of the Kolmogorov equations with respect to anisotropic SobolevSlobodeckii spaces. Compared to the setting in [24], we restrict ourselves to isotropic spaces and constant coefficients but, more generally, allow exponentially weighted spaces and possibly discontinuous killing rates.

To comprise all of the requirements, we state our question more precisely as follows: Under which conditions on the time-inhomogeneous Lévy process L, the possibly discontinuous killing rate $\kappa$, the source $f$ and initial condition $g$ is there a unique weak solution in an exponentially weighted Sobolev-Slobodeckii space of the Kolmogorov equation (1.1) that allows a stochastic representation of the form (1.2)?

To answer our question, we introduce in the next section the necessary notation and concepts. We use this framework first to characterize parabolicity of the Kolmogorov equation in terms of properties of the symbol in Theorem 3.3. Armed with this, we formulate our main result, the Feynman-Kac-type representation of the weak solution of the Kolmogorov equation (1.1) in Theorem 3.4. In Sect. 4, we find that a wide and interesting class of stochastic processes fall within the scope of this result. Analyzing its applications in Sect. 5 leads us from typical financial problems further to the characterization of purely probabilistic objects. Exploiting the advantages of Theorem 3.4 further, we return to its practical realization and implement a Galerkin scheme to solve (1.1) in Sect. 6. We find that thanks to Theorem 3.4, the solutions thus obtained correspond to option prices. With the numerical implementation at hand, we visualize and discuss the effect of killing rates of indicator type. Section 7 presents a robustness result for weak solutions that is required in our proof of Theorem 3.4 in Sect. 8. In this last section, we also identify desirable regularity properties of the solutions to the Kolmogorov equation. Appendix A provides two technical lemmas for the symbol and the operator, and Appendix B concludes with the proof of Theorem 3.3. 


\section{Preliminaries and notation}

In order to present the main result of the present article, we first introduce the underlying stochastic processes, the Kolmogorov equation with killing rate, its weak formulation as well as the solution spaces of our choice. We denote by $C_{0}^{\infty}\left(\mathbb{R}^{d}\right)$ the set of smooth real-valued functions with compact support in $\mathbb{R}^{d}$ and let

$$
\mathcal{F}(\varphi):=\int_{\mathbb{R}^{d}} \mathrm{e}^{i\langle\xi, x\rangle} \varphi(x) \mathrm{d} x
$$

be the Fourier transform of $\varphi \in C_{0}^{\infty}\left(\mathbb{R}^{d}\right)$ and $\mathcal{F}^{-1}$ its inverse.

Let a stochastic basis $\left(\Omega, \mathcal{F}_{T},\left(\mathcal{F}_{t}\right)_{0 \leq t \leq T}, P\right)$ be given and let $L$ be an $\mathbb{R}^{d}$-valued time-inhomogeneous Lévy process with characteristics $\left(b_{t}, \sigma_{t}, F_{t} ; h\right)_{t \geq 0}$. That is, $L$ has independent increments and for fixed $t \geq 0$, its characteristic function is given by

$$
E\left[\mathrm{e}^{i\left\langle\xi, L_{t}\right\rangle}\right]=\mathrm{e}^{-\int_{0}^{t} A_{s}(-i \xi) \mathrm{d} s} \quad \text { for every } \xi \in \mathbb{R}^{d},
$$

where for every $t \geq 0$ and $\xi \in \mathbb{R}^{d}$, the symbol of the process is defined as

$$
A_{t}(\xi):=\frac{1}{2}\left\langle\xi, \sigma_{t} \xi\right\rangle+i\left\langle\xi, b_{t}\right\rangle-\int_{\mathbb{R}^{d}}\left(\mathrm{e}^{-i\langle\xi, y\rangle}-1+i\langle\xi, h(y)\rangle\right) F_{t}(\mathrm{~d} y)
$$

Here, for every $s>0, \sigma_{s}$ is a symmetric, positive semi-definite $d \times d$-matrix, $b_{s} \in \mathbb{R}^{d}$ and $F_{s}$ is a Lévy measure, i.e., a positive Borel measure on $\mathbb{R}^{d}$ with $F_{s}(\{0\})=0$ and $\int_{\mathbb{R}^{d}}\left(|x|^{2} \wedge 1\right) F_{s}(\mathrm{~d} x)<\infty$. Moreover, $h$ is a truncation function, i.e., $h: \mathbb{R}^{d} \rightarrow \mathbb{R}^{d}$ is measurable with $\int_{\{|x|>1\}}|h(x)| F(\mathrm{~d} x)<\infty$ and $h(x)=x$ in a neighborhood of 0 . As usual, we assume the maps $s \mapsto \sigma_{s}, s \mapsto b_{s}$ and $s \mapsto \int\left(|x|^{2} \wedge 1\right) F_{s}(\mathrm{~d} x)$ to be Borel-measurable with, for every $T>0$,

$$
\int_{0}^{T}\left(\left|b_{s}\right|+\left\|\sigma_{s}\right\|_{\mathcal{M}(d \times d)}+\int_{\mathbb{R}^{d}}\left(|x|^{2} \wedge 1\right) F_{s}(\mathrm{~d} x)\right) \mathrm{d} s<\infty,
$$

where $\|\cdot\|_{\mathcal{M}(d \times d)}$ is a norm on the vector space of $d \times d$-matrices.

The Kolmogorov operator of the process $L$ is given by

$$
\begin{aligned}
\mathcal{A}_{t} \varphi(x):= & -\frac{1}{2} \sum_{j, k=1}^{d} \sigma_{t}^{j, k} \frac{\partial^{2} \varphi}{\partial x_{j} \partial x_{k}}(x)-\sum_{j=1}^{d} b_{t}^{j} \frac{\partial \varphi}{\partial x_{j}}(x) \\
& -\int_{\mathbb{R}^{d}}\left(\varphi(x+y)-\varphi(x)-\sum_{j=1}^{d} \frac{\partial \varphi}{\partial x_{j}}(x) h_{j}(y)\right) F_{t}(\mathrm{~d} y)
\end{aligned}
$$

for every $\varphi \in C_{0}^{\infty}\left(\mathbb{R}^{d}\right)$, where $h_{j}$ denotes the $j$ th component of the truncation function $h$. By some elementary manipulations, we obtain

$$
\mathcal{A}_{t} \varphi=\mathcal{F}^{-1}\left(A_{t} \mathcal{F}(\varphi)\right) \quad \text { for all } \varphi \in C_{0}^{\infty}\left(\mathbb{R}^{d}\right),
$$


which shows that the Kolmogorov operator $\mathcal{A}$ is a pseudo-differential operator with symbol $A$.

Following the classical way to define solution spaces of parabolic evolution equations, we introduce a Gelfand triplet $\left(V, H, V^{*}\right)$, which consists of a pair of separable Hilbert spaces $V$ and $H$ and the dual space $V^{*}$ of $V$ such that there exists a continuous embedding from $V$ into $H$. The scalar product in $H$ is denoted by $\langle\cdot, \cdot\rangle_{H}$ and the duality pairing is denoted by $\langle\cdot, \cdot\rangle_{V^{*} \times V}$. We denote by $L^{2}(0, T ; H)$ the space of weakly measurable functions $u:[0, T] \rightarrow H$ with $\int_{0}^{T}\|u(t)\|_{H}^{2} \mathrm{~d} t<\infty$ and by $\partial_{t} u$ the derivative of $u$ with respect to time in the distributional sense. The Sobolev space

$$
W^{1}(0, T ; V, H):=\left\{u \in L^{2}(0, T ; V) \mid \partial_{t} u \in L^{2}\left(0, T ; V^{*}\right)\right\}
$$

will serve as solution space for equation (1.1). For a more detailed introduction to the space $W^{1}(0, T ; V, H)$, which relies on the Bochner integral, we refer to [44, Sect. 24.2]. More information on Gelfand triplets can be found in [44, Sect. 17.1].

Usually, variational equations of a similar type as the heat equation are formulated with respect to Sobolev spaces, and thus are based on both $H^{1}$ and $L^{2}$. Since we include pure jump processes in our analysis, the operator (2.1) may be of fractional order. We therefore work with Sobolev-Slobodeckii spaces, which formalize the notion of a derivative of fractional order. Turning to a typical financial problem, we express the price of a call option as solution to a Kolmogorov equation of type (1.1). We then obtain $\kappa \equiv 0$ and $f \equiv 0$, while the initial condition is given by $g(x)=\left(S_{0} \mathrm{e}^{x}-K\right)^{+}$. We now have to realize that $g \notin L^{2}\left(\mathbb{R}^{d}\right)$ and we cannot use an $L^{2}$-based approach. The exponentially dampened function $x \mapsto g(x) \mathrm{e}^{\eta x}$, however, belongs to $L^{2}\left(\mathbb{R}^{d}\right)$ for every $\eta<-1$. Thus, in order to incorporate initial conditions that typically arise in financial problems, we allow an exponential weight. We may further increase the class of function spaces by a domain splitting argument; see Remark 5.2 below.

To make these considerations formally precise, we define the exponentially weighted Sobolev-Slobodeckii space $H_{\eta}^{\alpha}\left(\mathbb{R}^{d}\right)$ with an index $\alpha \geq 0$ and a weight $\eta \in \mathbb{R}^{d}$ as the completion of $C_{0}^{\infty}\left(\mathbb{R}^{d}\right)$ with respect to the norm $\|\cdot\|_{H_{\eta}^{\alpha}}$ given by

$$
\|\varphi\|_{H_{\eta}^{\alpha}}^{2}:=\int_{\mathbb{R}^{d}}(1+|\xi|)^{2 \alpha}|\mathcal{F}(\varphi)(\xi-i \eta)|^{2} \mathrm{~d} \xi .
$$

Observe that this is a separable Hilbert space. For $\eta=0$, the space $H_{0}^{\alpha}\left(\mathbb{R}^{d}\right)$ coincides with the Sobolev-Slobodeckii space $H^{\alpha}\left(\mathbb{R}^{d}\right)$ as it is defined e.g. in [44]. For $\alpha=0$ the space $H_{\eta}^{0}\left(\mathbb{R}^{d}\right)$ coincides with the weighted space

$$
L_{\eta}^{2}\left(\mathbb{R}^{d}\right):=\left\{u \in L_{\text {loc }}^{1}\left(\mathbb{R}^{d}\right) \mid x \mapsto u(x) \mathrm{e}^{\langle\eta, x\rangle} \in L^{2}\left(\mathbb{R}^{d}\right)\right\} .
$$

Furthermore, we denote the dual space of $H_{\eta}^{\alpha}\left(\mathbb{R}^{d}\right)$ by $H_{\eta}^{\alpha}\left(\mathbb{R}^{d}\right)^{*}$.

Let $a:[0, T] \times H_{\eta}^{\alpha}\left(\mathbb{R}^{d}\right) \times H_{\eta}^{\alpha}\left(\mathbb{R}^{d}\right) \rightarrow \mathbb{R}$ be a family $a=\left(a_{t}\right)_{t \in[0, T]}$ of bilinear forms that are measurable with respect to $t$ and let $\mathcal{A}=\left(\mathcal{A}_{t}\right)_{t \in[0, T]}$ with $\mathcal{A}_{t}: H_{\eta}^{\alpha}\left(\mathbb{R}^{d}\right) \rightarrow H_{\eta}^{\alpha}\left(\mathbb{R}^{d}\right)^{*}$ be a family of operators. We say a is associated with $\mathcal{A}$ if for every $t \in[0, T]$,

$$
\mathcal{A}_{t}(u)(v)=a_{t}(u, v) \quad \text { for all } u, v \in H_{\eta}^{\alpha}\left(\mathbb{R}^{d}\right) .
$$


Moreover, we call $A_{t}: \mathbb{R}^{d} \rightarrow \mathbb{C}$ the symbol associated with $\mathcal{A}_{t}$ if

$$
\mathcal{A}_{t}(\varphi)=\frac{1}{(2 \pi)^{d}} \int_{\mathbb{R}^{d}} \mathrm{e}^{-i\langle\xi, x\rangle} A_{t}(\xi) \mathcal{F}(\varphi)(\xi) \mathrm{d} \xi \quad \text { for all } \varphi \in C_{0}^{\infty}\left(\mathbb{R}^{d}\right) .
$$

We close the section with the weak formulation of the Kolmogorov equation (1.1).

Definition 2.1 Let $V=H_{\eta}^{\alpha}\left(\mathbb{R}^{d}\right)$ and $H=L_{\eta}^{2}\left(\mathbb{R}^{d}\right), \kappa:[0, T] \times \mathbb{R}^{d} \rightarrow \mathbb{R}$ measurable and bounded, $f \in L^{2}\left(0, T ; V^{*}\right)$ and $g \in H$. Then $u \in W^{1}(0, T ; V, H)$ is a weak solution of the Kolmogorov equation (1.1) if for almost every $t \in(0, T)$,

$$
\left\langle\partial_{t} u(t), v\right\rangle_{H}+a_{T-t}(u(t), v)+\left\langle\kappa_{T-t} u(t), v\right\rangle_{H}=\langle f(t) \mid v\rangle_{V^{*} \times V} \quad \text { for all } v \in V
$$

and $u(t)$ converges to $g$ in the norm of $H$ for $t \downarrow 0$.

\section{Main results}

Equipped with the necessary notations and concepts, we now focus on our main purpose, providing a Feynman-Kac formula linking weak solutions of PIDEs with killing rates to conditional expectations.

Following a classical way to prove existence and uniqueness of weak solutions of a parabolic equation, we verify continuity and a Gårding inequality of its bilinear form. We specify the notion of parabolicity accordingly and adapt it to our framework.

Definition 3.1 Let $\mathcal{A}$ be an operator associated with a bilinear form $a$. We say that $\mathcal{A}$, respectively $a$, is parabolic with respect to $H_{\eta}^{\alpha / 2}\left(\mathbb{R}^{d}\right), L_{\eta}^{2}\left(\mathbb{R}^{d}\right)$ if there exist constants $C, G>0, G^{\prime} \geq 0$ such that uniformly for all $t \in[0, T]$ and all $u, v \in H_{\eta}^{\alpha / 2}\left(\mathbb{R}^{d}\right)$, the following continuity and Gårding inequality hold:

$$
\begin{aligned}
\left|a_{t}(u, v)\right| & \leq C\|u\|_{H_{\eta}^{\alpha / 2}\left(\mathbb{R}^{d}\right)}\|v\|_{H_{\eta}^{\alpha / 2}\left(\mathbb{R}^{d}\right)}, \\
a_{t}(u, u) & \geq G\|u\|_{H_{\eta}^{\alpha / 2}\left(\mathbb{R}^{d}\right)}^{2}-G^{\prime}\|u\|_{L_{\eta}^{2}\left(\mathbb{R}^{d}\right)}^{2} .
\end{aligned}
$$

For $R \subset \mathbb{R}^{d}$, we say that the parabolicity of $\mathcal{A}$, respectively $a$, (with respect to $\left.\left(H_{\eta}^{\alpha / 2}\left(\mathbb{R}^{d}\right), L_{\eta}^{2}\left(\mathbb{R}^{d}\right)\right)_{\eta \in R}\right)$ is uniform in $[0, T] \times R$ if for all $u, v \in \bigcup_{\eta \in R} H_{\eta}^{\alpha / 2}\left(\mathbb{R}^{d}\right)$, the mapping $t \mapsto a_{t}(u, v)$ is piecewise continuous and the constants $C, G, G^{\prime}$ work for (3.1) and (3.2) simultaneously for all $\eta \in R$.

As highlighted in (2.2), the Kolmogorov operator of a time-inhomogeneous Lévy process is a pseudo-differential operator. Its symbol is explicitly known for various classes and in general is characterized by the exponent of the Lévy-Khinchine representation. Therefore, we express our main assumptions in terms of the symbol of the process. For Lévy processes with symbols $A$, it has been shown in [18, Theorem 3.1] that the corresponding bilinear form is parabolic with respect to $H^{\alpha / 2}\left(\mathbb{R}^{d}\right), L^{2}\left(\mathbb{R}^{d}\right)$ if and only if constants $C, G, G^{\prime}>0$ and $0 \leq \beta<\alpha$ exist such that for every $\xi \in \mathbb{R}^{d}$,

$$
|A(\xi)| \leq C(1+|\xi|)^{\alpha},
$$




$$
\Re(A(\xi)) \geq G(1+|\xi|)^{\alpha}-G^{\prime}(1+|\xi|)^{\beta} .
$$

We generalize this growth condition so as to render it suitable for the setting of timeinhomogeneous Lévy processes and weighted Sobolev-Slobodeckii spaces. We find that an extension of the bilinear form to such spaces corresponds to a shift of the symbol in the complex plane. Symbols can be extended to complex domains if the appropriate exponential moment condition is satisfied. In more detail, let $L$ be a timeinhomogeneous Lévy process. First notice that $L_{t}$ is infinitely divisible with Lévy measure $\widetilde{F}_{t}(\mathrm{~d} x):=\int_{0}^{t} F_{S}(\mathrm{~d} x) \mathrm{d} s$ for every $t \in[0, T]$, as has been shown by [15, Lemma 1]. Theorem 25.17 in [42] now implies that for all $\eta \in \mathbb{R}^{d}$,

$$
\int_{0}^{T} \int_{\{|x|>1\}} \mathrm{e}^{\langle\eta, x\rangle} F_{t}(\mathrm{~d} x) \mathrm{d} t<\infty
$$

is equivalent to the exponential moment condition $E\left[\mathrm{e}^{\left\langle\eta, L_{T}\right\rangle}\right]<\infty$ and

$$
E\left[\mathrm{e}^{\left\langle i \xi+\eta, L_{t}\right\rangle}\right]=\mathrm{e}^{-\int_{0}^{t} A_{s}(-\xi+i \eta) \mathrm{d} s} \quad \text { for all } \xi \in \mathbb{R}^{d} \text { and } t \geq 0 .
$$

We therefore formulate the conditions in terms of an exponential moment condition on the process and growth conditions on the symbol extended to a complex domain. It turns out that this complex domain can conveniently be chosen as a tensorized complex strip. More precisely, for a weight $\eta=\left(\eta_{1}, \ldots, \eta_{d}\right)$, let

$$
\begin{aligned}
& U_{\eta}:=\left\{z \in \mathbb{C}^{d} \mid \Im\left(z_{j}\right) \in\{0\} \cup \operatorname{sgn}\left(\eta_{j}\right)\left[0,\left|\eta_{j}\right|\right) \text { for } j=1, \ldots, d\right\}, \\
& R_{\eta}:=\operatorname{sgn}\left(\eta_{1}\right)\left[0,\left|\eta_{1}\right|\right] \times \cdots \times \operatorname{sgn}\left(\eta_{d}\right)\left[0,\left|\eta_{d}\right|\right] .
\end{aligned}
$$

From [42, Theorem 25.17], we also know that the complex set on which $A_{s}$ is definable is convex. Lemma 2.1(c) in [14] shows for the present setting that the map $z \mapsto A_{t}(z)$ has a continuous extension to the complex domain $\overline{U_{-\eta}}$ that is analytic in the interior $\stackrel{\circ}{U}_{-\eta}$.

We shall derive the main results using the following set of conditions.

Conditions 3.2 Fix a weight $\eta \in \mathbb{R}^{d}$ and index $\alpha \in(0,2]$, and let $A=\left(A_{t}\right)_{t \in[0, T]}$ be a symbol with extension to $\overline{U_{-\eta}}$.

(A1) (Exponential moment condition) If $L$ is a time-inhomogeneous Lévy process with symbol $A$, then for every $\eta^{\prime} \in R_{\eta}$ and $t \in[0, T]$,

$$
E\left[\mathrm{e}^{-\left\langle\eta^{\prime}, L_{t}\right\rangle}\right]<\infty .
$$

(A2) (Continuity condition) There exists a constant $C>0$ such that uniformly for all $\eta^{\prime} \in R_{\eta}$ and $t \in[0, T]$,

$$
\left|A_{t}\left(\xi-i \eta^{\prime}\right)\right| \leq C(1+|\xi|)^{\alpha} .
$$

(A3) (Gårding condition) There exist constants $G>0, G^{\prime} \geq 0$ and $0 \leq \beta<\alpha$ such that uniformly for all $\eta^{\prime} \in R_{\eta}$ and $t \in[0, T]$,

$$
\Re\left(A_{t}\left(\xi-i \eta^{\prime}\right)\right) \geq G(1+|\xi|)^{\alpha}-G^{\prime}(1+|\xi|)^{\beta} .
$$


(A4) For every fixed $\eta^{\prime} \in R_{\eta}$ and $\xi \in \mathbb{R}^{d}$, the mapping $t \mapsto A_{t}\left(\xi-i \eta^{\prime}\right)$ is piecewise continuous.

We say that $A$ has Sobolev index $\alpha$ uniformly in $[0, T] \times R_{\eta}$ if $A$ has an extension to $\overline{U_{-\eta}}$ that satisfies (A2)-(A4). If $A$ is the symbol of a process $L$, we also say $L$ has Sobolev index $\alpha$ uniformly in $[0, T] \times R_{\eta}$.

Conditions (A1)-(A4) are satisfied for a large set of processes, for instance tempered stable and normal inverse Gaussian processes as well as their timeinhomogeneous extensions. In Sect. 4, we look in detail at the verification of the conditions.

Notice that for $\eta=0$, we have $R_{0}=\{0\}$. Thus (A1) is trivially satisfied and (A2) and (A3) simplify accordingly. This case corresponds to the case of SobolevSlobodeckii spaces without weighting and is covered by the following results. If, moreover, the symbol is constant in time, (A4) is trivially satisfied and (A1)-(A4) reduce to (3.3) and (3.4). Conditions (A1)-(A3) were introduced in [14] to show existence and uniqueness of weak solutions of the related Kolmogorov equation (without killing rate) along with a Feynman-Kac-type formula with application to European option prices in time-inhomogeneous Lévy models. We additionally require (A4), which only imposes a mild technical restriction.

With our framework defined, let us now state our main results. First, Theorem 3.3 shows the equivalence between parabolicity with respect to weighted SobolevSlobodeckii spaces and the growth conditions (A2) and (A3), thereby generalizing the result for Sobolev-Slobodeckii spaces and conditions (3.3) and (3.4) to the present setting. The characterization of (uniform) parabolicity in terms of conditions on the symbol is interesting in its own right. It is, moreover, one of the key steps in our proof of Theorem 3.4 below, which establishes a Feynman-Kac-type representation.

Theorem 3.3 For $\eta \in \mathbb{R}^{d}$ and $\alpha \in(0,2]$, let L be a time-inhomogeneous Lévy process satisfying the exponential moment condition (A1). Then the following two assertions are equivalent:

(i) The Kolmogorov operator of $L$ is uniformly parabolic in $[0, T] \times R_{\eta}$ with respect to $\left(H_{\eta^{\prime}}^{\alpha / 2}\left(\mathbb{R}^{d}\right), L_{\eta^{\prime}}^{2}\left(\mathbb{R}^{d}\right)\right)_{\eta^{\prime} \in R_{\eta}}$.

(ii) L has Sobolev index $\alpha$ uniformly in $[0, T] \times R_{\eta}$.

The proof of Theorem 3.3 is given in Appendix B. There, we first give an interesting generalization of this result for operators and symbols that are not necessarily related to stochastic processes.

Assume (A1)-(A4). Then Theorem 3.3 shows in particular the parabolicity of the related bilinear form uniformly in time with respect to $H_{\eta}^{\alpha / 2}\left(\mathbb{R}^{d}\right)$ and $L_{\eta}^{2}\left(\mathbb{R}^{d}\right)$. Now the classical existence and uniqueness result, see for instance [45, Theorem 23.A], gives us that the Kolmogorov equation (1.1) has a unique weak solution $u$ in the space $W^{1}\left(0, T ; H_{\eta}^{\alpha / 2}\left(\mathbb{R}^{d}\right), L_{\eta}^{2}\left(\mathbb{R}^{d}\right)\right)$.

We now turn to the stochastic representation of this solution. For an integrable or nonnegative random variable $X$, we denote

$$
E_{0, x}[X]:=E_{x}[X], \quad E_{t, x}[X]:=E\left[X \mid L_{t}=x\right] \quad \text { for } t>0,
$$


where $x \mapsto E\left[X \mid L_{t}=x\right]$ is the factorization of the conditional expectation $E\left[X \mid L_{t}\right]$ and $E_{x}$ denotes the expectation with respect to the probability measure $P_{x}$ such that $P_{x}\left[L_{0}=x\right]=1$. Moreover, we denote by $\operatorname{supp}\left(L_{t}\right)$ the interior of the set $\left\{x \in \mathbb{R}^{d} \mid P\left[\left|L_{t}-x\right|<\epsilon\right]>0\right.$ for all $\left.\epsilon>0\right\}$.

Theorem 3.4 For $\eta \in \mathbb{R}^{d}$ and $\alpha \in(0,2]$, let L be an $\mathbb{R}^{d}$-valued time-inhomogeneous Lévy process with symbol $A=\left(A_{t}\right)_{t \in[0, T]}$ that satisfies (A1)-(A4). Then:

(i) For $\kappa:[0, T] \times \mathbb{R}^{d} \rightarrow \mathbb{R}$ measurable and bounded, $f \in L^{2}\left(0, T ; H_{\eta}^{\alpha / 2}\left(\mathbb{R}^{d}\right)^{*}\right)$ and $g \in L_{\eta}^{2}\left(\mathbb{R}^{d}\right)$, the Kolmogorov equation (1.1) has a unique weak solution $u$ in the space $W^{1}\left(0, T ; H_{\eta}^{\alpha / 2}\left(\mathbb{R}^{d}\right), L_{\eta}^{2}\left(\mathbb{R}^{d}\right)\right)$;

(ii) If additionally $f \in L^{2}\left(0, T ; H_{\eta}^{\ell}\left(\mathbb{R}^{d}\right)\right)$ for some $\ell \geq 0$ with $\ell>(d-\alpha) / 2$, then for every $t \in[0, T]$, we have (1.2) a.s. and for a.e. $x \in \operatorname{supp}\left(L_{1}\right)$,

$$
\begin{aligned}
& u(T-t, x) \\
& =E_{t, x}\left[g\left(L_{T}\right) \mathrm{e}^{-\int_{t}^{T} \kappa_{h}\left(L_{h}\right) \mathrm{d} h}+\int_{t}^{T} f\left(T-s, L_{s}\right) \mathrm{e}^{-\int_{t}^{s} \kappa_{h}\left(L_{h}\right) \mathrm{d} h} \mathrm{~d} s\right] .
\end{aligned}
$$

As we have already seen, part (i) of Theorem 3.4 follows from Theorem 3.3 and the classical existence and uniqueness result for solutions of parabolic equations. Part (ii) is considerably more involved, and Sect. 8 is devoted to its proof.

The major benefit of Theorem 3.4 for financial applications is that conditional expectations of the form (1.2) or (3.8), which naturally appear as financial quantities such as prices of derivatives, are now characterized by weak solutions of PIDEs. Therefore, the prices can be computed by numerically solving an equation of the form (1.1). To illustrate the method and the effect of killing rates, we present among other things an application to employee options in Sect. 5 and provide its Galerkin discretization in Sect. 6.

Theorem 3.4 furthermore shows a specific type of regularity of the conditional expectation (3.8). It is interesting to identify sufficient conditions for Hölder-continuity. Theorem 8.2 in [35] provides the appropriate Sobolev embedding result. Thus we obtain as an immediate consequence of Theorem 3.4 the following corollary.

Corollary 3.5 Under the assumptions and notations of Theorem 3.4 in the univariate case, i.e., for $d=1$, for $\alpha \in(1,2]$ and any fixed $t \in(0, T)$, the function $x \mapsto u(t, x)$ is $\lambda$-Hölder-continuous with $\lambda=\frac{\alpha-1}{2}$, i.e.,

$$
\sup _{x, y \in \mathbb{R}, x \neq y} \frac{|u(t, x)-u(t, y)|}{|x-y|^{\lambda}}<\infty .
$$

In particular, $x \mapsto u(t, x)$ is continuous and the equality (3.8) in Theorem 3.4 holds for every $x \in \operatorname{supp}\left(L_{1}\right)$. 


\section{Examples of classes of time-inhomogeneous Lévy processes}

Let us explore the nature of conditions (A1)-(A4) and show that they are satisfied for a wide class of processes. The conditions (A1)-(A4) naturally apply to processes that are specified through their symbols. Notice that the symbol is expressed in terms of the characteristics of the process. We exploit this in Proposition 4.8 to establish concrete accessible conditions for real valued time-inhomogeneous pure jump Lévy processes with absolutely continuous Lévy measures, while Proposition 4.3 treats time-inhomogeneous multivariate jump-diffusions.

We should, however, realize that conditions (A1)-(A4) are not satisfied by all Lévy processes. On the one hand, continuity (A2) and the Gårding condition (A3) have implications for the distributional properties of the process.

Remark 4.1 Fix $\eta \in \mathbb{R}^{d}$ and $\alpha \in(0,2]$, and let $L$ be a time-inhomogeneous Lévy process with symbol $A=\left(A_{t}\right)_{t \geq 0}$. If a Gårding condition (A3) is satisfied for a weight $\eta$ and index $\alpha$, then there exist $C_{1}, C_{2}>0$ such that uniformly for all $\eta^{\prime} \in R_{\eta}$ and $0 \leq s \leq t \leq T$,

$$
\left|\mathrm{e}^{-\int_{s}^{t} A_{u}\left(\xi-i \eta^{\prime}\right) \mathrm{d} u}\right| \leq C_{1} \mathrm{e}^{-(t-s) C_{2}|\xi|^{\alpha}} .
$$

In particular, (A3) implies for every $t \in(0, T]$ that the distribution of $L_{t}$ has a smooth Lebesgue density.

On the other hand, continuity (A2) and the Garding condition (A3) relate to the path behavior of the process: If a Lévy process with symbol $A$ satisfies (A2) and (A3) for $\alpha \in(0,2)$ and $\eta=0$, then $\alpha$ is its Blumenthal-Getoor index, as shown in [18, Theorem 4.1]. Hence, every pure jump Lévy process satisfying assumptions (A2) and (A3) has infinite jump activity. On this basis, we may for instance conclude that compound Poisson processes do not satisfy (A3).

Variance gamma processes have Blumenthal-Getoor index 0 and thus do not satisfy both (A2) and (A3), as noticed in part (iv) of Example 4.1 in [18]. However, pure jump Lévy processes can be approximated by a sequence of Lévy jump-diffusion processes with nonzero Brownian part. This can always be achieved by adding a diffusion part and letting its volatility coefficient tend to zero. Thus Example 4.4 shows that pure jump Lévy processes can be approximated by Lévy processes for which (A1)-(A3) are satisfied for weight $\eta=0$ and index $\alpha=2$. The authors of [4] provide a sequence with better approximation properties for Monte Carlo techniques, which could be exploited further in the present context.

Before continuing the discussion on the validity of conditions (A1)-(A4) for other classes of processes, we observe the following.

Remark 4.2 For $\eta \in \mathbb{R}^{d}$ and $\alpha \in(0,2]$, let $A$ be the symbol of a Lévy process that satisfies the exponential moment condition (A1). By virtue of Lemma A.1 in Appendix $\mathrm{A}$ and the continuity of symbols of Lévy processes (as mappings from $\mathbb{R}^{d}$ to $\mathbb{C}$ ), the validity of the continuity condition (A2) for $A$ is equivalent to the following asymptotic condition: For every $N>0$, there exists a constant $G>0$ such that 
for every $\eta^{\prime} \in R_{\eta}$,

$$
\Re\left(A\left(\xi-i \eta^{\prime}\right)\right) \geq G|\xi|^{\alpha}-A\left(i \eta^{\prime}\right) \quad \text { for every } \xi \in \mathbb{R}^{d} \text { such that }|\xi|>N \text {. }
$$

We devote the remainder of this section to providing sufficient conditions for the validity of conditions (A1)-(A4) for time-inhomogeneous jump-diffusions, for pure jump Lévy processes, and for time-inhomogeneous processes.

\subsection{Jump-diffusions}

For time-inhomogeneous Lévy jump-diffusion processes, we find that conditions (A1)-(A4) are satisfied under remarkably weak conditions.

Proposition 4.3 Fix some $\eta \in \mathbb{R}^{d}$. Let $L$ be a time-inhomogeneous Lévy process with characteristics $\left(b_{t}, \sigma_{t}, F_{t} ; h\right)_{0 \leq t \leq T}$ such that

$$
\begin{gathered}
\sup _{t \in[0, T]} \int_{\{|x|>1\}} \mathrm{e}^{-\left\langle\eta^{\prime}, x\right\rangle} F_{t}(\mathrm{~d} x)<\infty \quad \text { for every } \eta^{\prime} \in R_{\eta}, \\
\sup _{t \in[0, T]}\left\{\left|b_{t}\right|+\left\|\sigma_{t}^{-1}\right\|+\left\|\sigma_{t}\right\|+\int_{\mathbb{R}^{d}}\left(|x|^{2} \wedge 1\right) F_{t}(\mathrm{~d} x)\right\}<\infty .
\end{gathered}
$$

Then (A1)-(A3) are satisfied for the weight $\eta$ and index $\alpha=2$.

Proof Due to the equivalence of (3.5) and the exponential moment condition, (4.2) implies (A1). Observing that

$$
\begin{aligned}
\Re\left(A_{t}\left(\xi-i \eta^{\prime}\right)\right)= & \left\langle b_{t}, \eta^{\prime}\right\rangle+\frac{1}{2}\left\langle\eta^{\prime}, \sigma_{t} \eta^{\prime}\right\rangle+\int_{\mathbb{R}^{d}}\left(\left(\left\langle h(x), \eta^{\prime}\right\rangle-1\right) \mathrm{e}^{-\left\langle\eta^{\prime}, x\right\rangle}-1\right) F_{t}(\mathrm{~d} x) \\
& +\frac{1}{2}\left\langle\xi, \sigma_{t} \xi\right\rangle+\int_{\mathbb{R}^{d}}(\cos \langle\xi, x\rangle-1) F_{t}(\mathrm{~d} x)
\end{aligned}
$$

and $\int_{\mathbb{R}^{d}}(\cos \langle\xi, x\rangle-1) F_{t}(\mathrm{~d} x) \geq 0$, the inequalities (4.2) and (4.3) yield the Gårding condition (A3) with $\alpha=2$. Similarly, the inequalities (4.2), (4.3) and

$$
\Im\left(A_{t}\left(\xi-i \eta^{\prime}\right)\right)=\left\langle b_{t}^{-\eta^{\prime}}, \xi\right\rangle+\int_{\mathbb{R}^{d}}(\sin \langle\xi, x\rangle-\langle\xi, h(x)\rangle) \mathrm{e}^{-\left\langle\eta^{\prime}, x\right\rangle} F_{t}(\mathrm{~d} x),
$$

where $b_{t}^{-\eta^{\prime}}=b_{t}+\sigma_{t} \eta^{\prime}+\int_{\mathbb{R}^{d}}\left(\mathrm{e}^{\left\langle\eta^{\prime}, y\right\rangle}-1\right) h(y) F_{t}(\mathrm{~d} y)$ as defined in Lemma A.1 in Appendix A, yield the continuity condition (A2), which concludes the proof.

For Lévy jump-diffusion processes, the conditions simplify considerably.

Example 4.4 (Multivariate Lévy processes with Brownian part) Fix $\eta \in \mathbb{R}^{d}$ and let $L$ be an $\mathbb{R}^{d}$-valued Lévy processes with characteristics $(b, \sigma, F ; h)$ such that $\sigma$ is a positive definite matrix and the Lévy measure $F$ satisfies $\int_{\{|x|>1\}} \mathrm{e}^{-\eta x} F_{t}(\mathrm{~d} x)<\infty$. Then (A1)-(A3) hold for the weight $\eta \in \mathbb{R}^{d}$ and index $\alpha=2$. 
In order to verify those assumptions of Proposition 4.3 that concern the pure jump part of the process, it suffices to consider the pure jump processes separately, as the following lemma shows.

Lemma 4.5 For $j=1,2$, let $L^{j}$ be two stochastically independent time-inhomogeneous Lévy processes with symbols $A^{j}$ such that (A1)-(A4) are satisfied for the same weight $\eta \in \mathbb{R}^{d}$ and the possibly different indices $\alpha^{j} \in(0,2]$. Then the sum $L:=L^{1}+L^{2}$ is a time-inhomogeneous Lévy process with symbol $A:=A^{1}+A^{2}$, and (A1)-(A4) are satisfied for the weight $\eta$ and index $\alpha:=\max \left(\alpha^{1}, \alpha^{2}\right)$.

Lemma 4.5 generalizes Remark 4.1 in [18] to the case where $\eta \neq 0$, and we omit its elementary proof.

\subsection{Pure jump Lévy processes and operators of fractional order}

We now consider a class of multivariate processes which frequently occur in finance and whose symbol is explicitly given.

Example 4.6 (Multivariate normal inverse Gaussian (NIG) processes) Let $L$ be an $\mathbb{R}^{d}$-valued NIG process, which is defined as a Lévy process such that we have $L_{1}=\left(L_{1}^{1}, \ldots, L_{1}^{d}\right) \sim \operatorname{NIG}_{d}(\tilde{\alpha}, \beta, \delta, \mu, \Delta)$ for parameters $\tilde{\alpha}, \delta \geq 0, \beta, \mu \in \mathbb{R}^{d}$ and a symmetric positive definite matrix $\Delta \in \mathbb{R}^{d \times d}$ with $\tilde{\alpha}^{2}>\langle\beta, \Delta \beta\rangle$. The symbol of $L$ is given by

$$
A(u)=i\langle u, \mu\rangle-\delta\left(\sqrt{\tilde{\alpha}^{2}-\langle\beta, \Delta \beta\rangle}-\sqrt{\tilde{\alpha}^{2}-\langle\beta+i u, \Delta(\beta+i u)\rangle}\right),
$$

where we denote by $\langle\cdot, \cdot\rangle$ the (real) scalar product $\left\langle z, z^{\prime}\right\rangle=\sum_{j=1}^{d} z_{j} z_{j}^{\prime}$ for $z \in \mathbb{C}^{d}$. Compare e.g. [21, Eq. (2.3)].

Assumptions (A1)-(A3) are satisfied for the index $\alpha=1$ and every $\eta \in \mathbb{R}^{d}$ such that $\tilde{\alpha}^{2}>\left\langle\beta+\eta^{\prime}, \Delta\left(\beta+\eta^{\prime}\right)\right\rangle$ for all $\eta^{\prime} \in R_{\eta}$. This is in particular the case if

$$
\|\beta\|^{2}+\|\eta\|^{2} \leq \tilde{\alpha}^{2} /\|\Delta\| .
$$

To summarize, if the parameters of $L$ satisfy (4.4), conditions (A1)-(A4) are satisfied for the weight $\eta$ and Sobolev index 1 .

Since pure jump Lévy processes can be defined through a Lévy measure and a constant drift, we are interested in finding conditions on both the Lévy measure and the drift that imply conditions (A1)-(A4). Let us address this issue for real-valued timehomogeneous pure jump Lévy processes whose Lévy measure is absolutely continuous. For this class, we generalize [18, Proposition 4.2] to time-inhomogeneity and weights $\eta \neq 0$. Thereby we obtain explicit conditions on the characteristics that imply conditions (A1)-(A4).

Conditions 4.7 Fix $\eta \in \mathbb{R}$ and $\alpha \in(0,2]$, and let $L$ be a real-valued timeinhomogeneous Lévy process with characteristics $\left(b_{t}, \sigma_{t}, F_{t} ; h\right)_{t \geq 0}$. 
(F1) $\int_{0}^{T} \int_{\{|x|>1\}} \mathrm{e}^{-\eta x} F_{t}(\mathrm{~d} x) \mathrm{d} t<\infty$.

(F2) $F_{t}$ is absolutely continuous for every $t \in[0, T]$ with $F_{t}(\mathrm{~d} x)=f_{t}(x) \mathrm{d} x$. In order to formulate the following conditions, we denote the symmetric and antisymmetric parts of the density by $f_{t}^{\mathrm{sym}}(x):=\left(f_{t}(x)+f_{t}(-x)\right) / 2$ and $f_{t}^{\text {asym }}(x):=f_{t}(x)-f_{t}^{\text {sym }}(x)$.

(F3) There exist constants $C_{1}, C_{2}, \epsilon>0$ and $0 \leq \beta<\alpha<2$ and a function $g:[0, T] \times[-\epsilon, \epsilon] \rightarrow \mathbb{R}$ such that uniformly for all $t \in[0, T]$,

$$
f_{t}^{\mathrm{sym}}(x) \leq \frac{C_{1}}{|x|^{1+\alpha}}+g(t, x) \quad \text { and } \quad|g(t, x)| \leq \frac{C_{2}}{|x|^{1+\beta}} \quad \text { for all }|x|<\epsilon .
$$

(F4) If $\alpha=1$, there exist constants $C_{3}, \epsilon>0$ and $\beta \in(0,1]$ such that uniformly for all $t \in[0, T]$,

$$
\left|f_{t}^{\text {asym }}(x)\right| \leq \frac{C_{3}}{|x|^{1+\beta}} \quad \text { for all }|x|<\epsilon .
$$

If $\alpha<1$, then the inequality (4.5) holds for some $\beta \in[0, \alpha]$, and moreover, $b_{t}=\int h(x) F_{t}(\mathrm{~d} x)$ for every $t \in[0, T]$.

Proposition 4.8 Let $L$ be a real-valued time-inhomogeneous pure jump Lévy process with characteristics $\left(b_{t}, 0, F_{t}\right)_{t \geq 0}$. Then:

(i) Condition (F1) is equivalent to (A1).

(ii) Conditions (F1)-(F3) imply (A1) and (A3).

(iii) Conditions (F1)-(F4) imply (A1)-(A3).

Proof (i) Since $d=1$, we have $R_{\eta}=\operatorname{sgn}(\eta)[0,|\eta|]$, and part (i) directly follows from Theorem 25.17 in [42].

(ii) We denote $f_{t, \eta^{\prime}}(x):=\mathrm{e}^{\eta^{\prime} x} f_{t}(x)$ and the symmetric and antisymmetric parts $f_{t, \eta^{\prime}}^{\mathrm{sym}}(x):=\left(f_{t, \eta^{\prime}}(x)+f_{t, \eta^{\prime}}(-x)\right) / 2$ and $f_{t, \eta^{\prime}}^{\text {asym }}:=f_{t, \eta^{\prime}}-f_{t, \eta^{\prime}}^{\text {sym }}$. Then the elementary identity $a b+c d=(a+c)(b+d) / 2+(a-c)(b-d) / 2$ yields

$$
f_{t, \eta^{\prime}}^{\mathrm{sym}}(x)=\cosh \left(\eta^{\prime} x\right) f_{t, \eta^{\prime}}^{\mathrm{sym}}(x)+\sinh \left(\eta^{\prime} x\right) f_{t, \eta^{\prime}}^{\mathrm{asym}}(x) .
$$

We notice that there exists a constant $c>0$ such that

$$
\cosh \left(\eta^{\prime} x\right) \geq \mathrm{e}^{-\eta^{\prime} \epsilon} \quad \text { and } \quad\left|\sinh \left(\eta^{\prime} x\right)\right| \leq c|x| \quad \text { for every }|x|<\epsilon .
$$

Moreover, since $f \geq 0$, the triangle inequality implies $\left|f_{t, \eta^{\prime}}^{\text {asym }}(x)\right| \leq f_{t, \eta^{\prime}}^{\mathrm{sym}}(x)$ for every $(t, x) \in[0, T] \times \mathbb{R}$. This shows that condition (F3) also remains valid when we replace $f_{t}^{\text {sym }}$ by $f_{t, \eta^{\prime}}^{\text {sym }}$ for every $\eta^{\prime} \in R_{\eta}$. Now part (ii) follows from inequality (4.16) in the proof of Proposition 4.2 in [18].

(iii) Along the same lines as in the proof of part (ii), we observe that

$$
f_{t, \eta^{\prime}}^{\text {asym }}(x)=\sinh \left(\eta^{\prime} x\right) f_{t, \eta^{\prime}}^{\text {sym }}(x)+\cosh \left(\eta^{\prime} x\right) f_{t, \eta^{\prime}}^{\text {asym }}(x) .
$$

Thus the validity of condition (F4) also remains valid when replacing $f_{t}^{\text {asym }}$ by $f_{t, \eta^{\prime}}^{\text {asym }}$. Then Proposition 4.2 in [18] shows the assertion for $\alpha=1$. 
For $\alpha<1$, we have $b_{t}=\int h(x) F_{t}(\mathrm{~d} x)$. According to Lemma A.1 and using the notation therein, $b_{t}^{-\eta^{\prime}}=\int h(x) F_{t}^{-\eta^{\prime}}(\mathrm{d} x)$. Hence, for all $t \in[0, T]$ and $\eta^{\prime} \in R_{\eta}$,

$$
\Im\left(A\left(\xi-i \eta^{\prime}\right)\right)=\int_{\mathbb{R}} \sin (\xi x) \mathrm{e}^{-\eta^{\prime} x} F_{t}(\mathrm{~d} x) .
$$

Estimating the real part of $A\left(\xi-i \eta^{\prime}\right)$ along the same lines as in the proof of Proposition 4.2 in [18], we obtain the continuity condition (A2).

We now apply Proposition 4.8 to a concrete class of processes which is frequently used to model asset prices.

Example 4.9 (Univariate generalized tempered stable Lévy processes) Let $L$ be a generalized tempered stable Lévy process with parameters $C_{-}, C_{+} \geq 0$ such that $C_{-}+C_{+}>0$ and $G, M>0$ and $Y_{-}, Y_{+}<2$. That is, $L$ is a pure jump Lévy process with characteristic triplet $\left(b, 0, F^{\text {temp }} ; h\right)$ with $F^{\text {temp }}(\mathrm{d} x)=f^{\text {temp }}(x) \mathrm{d} x$, where

$$
f^{\text {temp }}(x)= \begin{cases}\frac{C_{-}}{|x|^{1+Y_{-}}} \mathrm{e}^{G x} & \text { for } x<0, \\ \frac{C_{+}}{|x|^{1+Y_{+}}} \mathrm{e}^{-M x} & \text { for } x \geq 0 .\end{cases}
$$

For $C_{ \pm}=0$, we set $Y_{ \pm}:=0$. For $C=C_{-}=C_{+}$and $Y=Y_{-}=Y_{+}$, this class is known as CGMY, after Carr, Geman, Madan and Yor. Tempered stable processes are also referred to as Koponen and $\mathrm{KoBoL}$ in the literature; see e.g. [8]. For the general setting, see for instance [39].

Thanks to Proposition 4.8, the conditions (A1)-(A3) are satisfied for every weight $\eta \in(-G, M)$ and Sobolev index $\alpha:=\max \left\{Y_{+}, Y_{-}\right\}$in each of the following cases:

(i) $\alpha=\max \left\{Y_{+}, Y_{-}\right\}>1$,

(ii) $Y:=Y_{-}=Y_{+}=1$ and $C_{-}=C_{+}$,

(iii) $0<\alpha=\max \left\{Y_{+}, Y_{-}\right\}<1$ and $b=\int h(x) F(\mathrm{~d} x)$.

Further examples are considered in [18] for the case $\eta=0$. There, Examples 4.54.7 give conditions on the parameters that imply conditions (A2) and (A3) for generalized Student- $t$, Cauchy, generalized hyperbolic and stable processes. Moreover, again for the case $\eta=0,[18$, Sect. 4.3] provides a sufficient tail condition on the Lévy measure for (A2) and (A3) to hold.

\subsection{Time-inhomogeneous processes}

When modeling with Lévy processes in finance, we often need to consider the larger class of time-inhomogeneous Lévy processes because their flexibility in time leads to a considerably better fit to the time evolution of data. We therefore propose two construction principles that lead to parametric families of time-inhomogeneous processes satisfying (A1)-(A4).

First, we find it natural to define a family of time-inhomogeneous Lévy processes by inserting time-dependent parameters into a given parametric class of Lévy processes. For this class, it turns out to be straightforward to show the following result. 
Lemma 4.10 Let $\mathcal{P} \subset \mathbb{R}^{D}$ for some $D>0$ and $(A(p, \cdot))_{p \in \mathcal{P}}$ a parametrized family of symbols. Fix some $\eta \in \mathbb{R}^{d}$ and some $\alpha \in(0,2]$. Let (A2) and (A3) be satisfied for $A$, uniformly for all $p \in \mathcal{P}$. If $t \mapsto p(t)$ is measurable, then (A2) and (A3) are satisfied for

$$
A_{t}(\xi):=A(p(t), \xi) \quad \text { for } t \in[0, T] \text { and } \xi \in U_{-\eta} .
$$

If, moreover, $(p, \xi) \mapsto A(p, \xi)$ is continuous and $t \mapsto p(t)$ is piecewise continuous, then $\left(A_{t}\right)_{t \geq 0}$ is the symbol of a time-inhomogeneous Lévy process $L^{\prime}$ and also satisfies (A4). If additionally (A1) is satisfied for $L$, then it is also satisfied for $L^{\prime}$.

For $p(t)$ we can for instance choose a vector of piecewise constant parameters, so as to incorporate different short-, mid- and long-term behavior.

As another natural construction, let us consider stochastic integrals of deterministic functions with respect to Lévy processes. Let $L$ be an $\mathbb{R}^{d}$-valued Lévy process whose Lévy measure $F$ satisfies $\int_{\{|x|>1\}}|x| F(\mathrm{~d} x)<\infty$, i.e., it is a special semimartingale, and we denote its characteristics $(b, c, F ; h)$ with $h$ chosen as identity. Moreover, let $f:[0, \infty) \rightarrow \mathbb{R}^{n \times d}$ be a measurable function such that there exist constants $0<f_{*}, f^{*}$ with

$$
\sup _{0 \leq t \leq T}\left\|\left(f(t) f(t)^{\top}\right)^{-1}\right\|^{1 / 2} \leq f_{*}^{-1} \quad \text { and } \quad \sup _{0 \leq t \leq T}\left\|f(t) f(t)^{\top}\right\|^{1 / 2} \leq f^{*},
$$

where $\|\cdot\|$ denotes the spectral norm. Then

$$
X_{t}:=f \cdot L_{t}:=\int_{0}^{t} f(s) \mathrm{d} L_{s}:=\left(\sum_{k=1}^{d} \int_{0}^{t} f^{j k}(s) \mathrm{d} L_{s}^{k}\right)_{j \leq d}
$$

defines an $\mathbb{R}^{n}$-valued semimartingale. Standard arguments from semimartingale theory show that the symbol $A^{X}$ of $X$ is given by

$$
A_{t}^{X}(\xi)=A\left(f(t)^{\top} \xi\right) \quad \text { for all } \xi \in \mathbb{R}^{n},
$$

and the Lévy measure $F^{X}$ of $X$ is of the form $F_{t}^{X}(B)=\int_{\mathbb{R}^{d}} \mathbb{1}_{B}(f(t) x) F(\mathrm{~d} x)$ for $B \in \mathcal{B}\left(\mathbb{R}^{d} \backslash\{0\}\right)$. Then, by the continuity of Lévy symbols and Lemma A.1, we obtain the following assertion on the validity of conditions (A1)-(A4).

Remark 4.11 Fix some $\rho>0, \eta^{X} \in \mathbb{R}^{d}$ with $\left|\eta^{X}\right| \leq \frac{\rho}{f^{*}}$ and some $\alpha>0$. If $E\left[\mathrm{e}^{\rho\left|L_{t}\right|}\right]<\infty$ for some $t>0$, then $X$ satisfies the exponential moment condition (A1) for the weight $\eta^{X}$. If additionally $A$ satisfies (A2) and (A3) for every weight $\eta \in \mathbb{R}^{d}$ with $|\eta| \leq \rho$ and index $\alpha>0$, then (A2) and (A3) hold for $A^{X}$ with the same index $\alpha$ and weight $\eta^{X}$. Moreover, if (A4) holds for $A$, it is also satisfied by $A^{X}$.

\section{Applications}

Having convinced ourselves that it is a wide and interesting class of stochastic processes for which Theorem 3.4 links conditional expectations with weak solutions of 
PIDEs, let us now explore the virtues of the result for applications. Starting with pricing problems in finance, where discontinuous killing rates arise naturally, we furthermore find that indicator-type killing rates also help us to characterize interesting probabilistic objects. In all these applications, the driving process $L$ can be chosen freely and we may employ jump-diffusions or pure jump processes. The latter are intensely used in finance. Examples are NIG and generalized tempered stable processes, which we have shown to satisfy the assumptions of Theorem 3.4.

\subsection{Employee options}

We propose a class of employee options that flexibly reward the management board according to the performance of the corporation's stock price. Financial instruments used in this context are called employee stock options and often are based on European call options. Thus the reward depends on the level of the stock at specific points in time. Shareholders, however, are typically interested in the performance of the stock during the whole period. They mean to support management decisions that push the stock price constantly to a high level. Moreover, it is arguably fairer to choose the reward according to the performance of the stock value as relative to the market evolution.

To make this formally precise, denote by $S$ the $d$-dimensional stochastic process that models the stock of the company and $d-1$ reference assets. Let $G: \mathbb{R}^{d} \rightarrow \mathbb{R}$ be a payout profile and $\kappa:[0, T] \times \mathbb{R}^{d} \rightarrow \mathbb{R}$ a reward rate function. For $\kappa<0$, the reward turns into a penalty. Moreover, we include a continuously paid salary by the salary function $f:[0, T] \times \mathbb{R}^{d} \rightarrow \mathbb{R}$. At maturity $T$, the employee obtains the payout

$$
G\left(S_{T}\right) \mathrm{e}^{\int_{0}^{T} \kappa_{h}\left(S_{h}\right) \mathrm{d} h},
$$

in addition to the (integrated) salary

$$
f\left(t, S_{t}\right) \mathrm{e}^{\int_{0}^{t} \kappa_{h}\left(S_{h}\right) \mathrm{d} h} \mathrm{~d} t
$$

which is paid at each instant $t \in[0, T]$. Thus, the payout profile $G$ may depend on the level of the stock and the reference assets. The reward rate and the salary may additionally be time-dependent. Note that our analysis allows us to incorporate discontinuities in the reward rate. Thus threshold- and indicator-type reward functions are allowed, which is a natural choice. Indicator-type killing rates for instance play the role of instantaneous rewards or penalties for stock price levels in a specified domain.

We further use the following notation. For $x=\left(x_{1}, \ldots, x_{d}\right) \in \mathbb{R}^{d}$, denote $\mathrm{e}^{x}:=\left(\mathrm{e}^{x_{1}}, \ldots, \mathrm{e}^{x_{d}}\right), \widetilde{G}(x):=G\left(\mathrm{e}^{x}\right), \tilde{\kappa}_{t}(x):=-\kappa_{t}\left(\mathrm{e}^{x}\right), \tilde{f}(t, x):=f\left(T-t, \mathrm{e}^{x}\right)$ for all $t \in[0, T]$. We now assume the interest rate $\left(r_{t}\right)_{t \geq 0}$ to be deterministic, measurable and bounded. We model $S=\left(S_{0}^{1} \mathrm{e}^{L^{1}}, \ldots, S_{0}^{d} \mathrm{e}^{L^{d}}\right)$ by a time-inhomogeneous Lévy process $L$ with local characteristics $(b, c, F ; h)$ such that the no-arbitrage condition

$$
b_{t}^{i}=r_{t}-\frac{1}{2} c_{t}^{i i}-\int_{\mathbb{R}}\left(e^{x_{i}}-1-h_{i}(x)\right) F_{t}(\mathrm{~d} x) \quad \text { for every } i=1, \ldots, d
$$

is satisfied, where $h_{i}$ is the $i$ th component of the truncation function $h$. 
The following assertion shows that the fair price of the employee option specified by (5.1) and (5.2) can be computed by solving the related Kolmogorov PIDE. The result is an immediate consequence of Theorem 3.4.

Corollary 5.1 Let $\eta \in \mathbb{R}^{d}$ and $\alpha \in(0,2]$ be such that $\widetilde{G} \in L_{\eta}^{2}\left(\mathbb{R}^{d}\right)$ and assume that the time-inhomogeneous Lévy process $L$ satisfies (5.3) and conditions (A1)-(A4). Denoting $x=\log S_{0}$, the fair price

$$
u(T, x):=E_{x}\left[\widetilde{G}\left(L_{T}\right) \mathrm{e}^{\int_{0}^{T}\left(\tilde{\kappa}_{h}\left(L_{h}\right)-r_{h}\right) \mathrm{d} h}+\int_{0}^{T} \tilde{f}\left(T-s, L_{s}\right) \mathrm{e}^{\int_{0}^{s}\left(\tilde{\kappa}_{h}\left(L_{h}\right)-r_{h}\right) \mathrm{d} h} \mathrm{~d} s\right]
$$

of the employee option with payout profile (5.1), (5.2) is given by the unique weak solution $u \in W^{1}\left(0, T ; H_{\eta}^{\alpha / 2}\left(\mathbb{R}^{d}\right), L_{\eta}^{2}\left(\mathbb{R}^{d}\right)\right)$ of

$$
\dot{u}+\mathcal{A}_{T-t} u+\tilde{\kappa}_{T-t} u=-\tilde{f}, \quad u(0)=\widetilde{G} .
$$

See Sect. 6 for a numerical implementation of (5.4).

\subsection{Lévy-driven short rate models}

Lévy-driven term structure models were first introduced in [16]. Here, we consider a short rate of the form

$$
r_{t}:=r\left(t, L_{t}\right)
$$

with an $\mathbb{R}^{d}$-valued time-inhomogeneous Lévy process $L$ and a measurable bounded interest rate function $r:[0, T] \times \mathbb{R}^{d} \rightarrow \mathbb{R}$. We allow discontinuities in the function $r$ and thus thresholds in the factor model (5.5).

At maturity, the holder of a zero-coupon bond receives one unit of currency. In accordance with the no-arbitrage principle, the time- $t$ value of the zero-coupon bond with maturity $0 \leq t \leq T$ is modeled by

$$
P(t, T):=E\left[\mathrm{e}^{-\int_{t}^{T} r_{h} \mathrm{~d} h} \mid \mathcal{F}_{t}\right]
$$

Translating this conditional expectation formally into an evolution problem of the form (1.1), we obtain $g(x) \equiv 1$ as initial condition. We now have to realize that there is no weight $\eta \in \mathbb{R}^{d}$ such that $x \mapsto \mathrm{e}^{\langle\eta, x\rangle} \in L^{2}\left(\mathbb{R}^{d}\right)$. We therefore split the initial condition into summands that each lie in a weighted $L^{2}$-space. In the one-dimensional case, for example, we have $g=\mathbb{1}_{(-\infty, 0]}+\mathbb{1}_{(0, \infty)}$, where $1_{(-\infty, 0]} \in L_{\eta^{-}}^{2}(\mathbb{R})$ for every $\eta^{-}>0$ and $1_{(0, \infty)} \in L_{\eta^{+}}^{2}(\mathbb{R})$ for every $\eta^{+}<0$.

Remark 5.2 We split the given function $g: \mathbb{R}^{d} \rightarrow \mathbb{R}$ into $2^{d}$ summands $g^{j}$ that are supported in the $2^{d}$ orthants. To be precise, for $j=1, \ldots, 2^{d}$, let $p^{j}:=\left(p_{1}^{j}, \ldots, p_{d}^{j}\right)$ with $p_{i}^{j} \in\{-1,1\}$ for the $2^{d}$ different possible configurations and let

$$
O^{j}:=\left\{\left(x_{1}, \ldots, x_{d}\right) \in \mathbb{R}^{d} \mid p_{i}^{j} x_{i} \geq 0 \text { for all } i=1, \ldots, d\right\} .
$$


By linearity of the expectation, respectively of the PIDE, the problem can be split additively into $2^{d}$ separate problems. If for each of the summands $g^{j}$, a weight $\eta^{j} \in \mathbb{R}^{d}$ exists such that $g^{j} \in L_{\eta^{j}}^{2}\left(\mathbb{R}^{d}\right)$, then the results of Theorem 3.4 can be applied to each problem with initial condition $g^{j}$ separately.

As in Remark 5.2, we split the unity as $1 \equiv g(x)=\sum_{j=1}^{2^{d}} \mathbb{1}_{O^{j}}(x)$ a.e. with the distinct orthants $O^{j}$ of $\mathbb{R}^{d}$ given by (5.7). For each $j$, we choose

$$
\eta^{j}:=-\epsilon d^{-1 / 2} p^{j}
$$

so that $\mathbb{1}_{O^{j}} \mathrm{e}^{\left\langle\eta^{j}, \cdot\right\rangle} \in L^{2}\left(\mathbb{R}^{d}\right)$. If the distribution of $L_{T}$ has a Lebesgue density, we may rewrite (5.6) as

$$
u(T-t, x)=\sum_{j=1}^{2^{d}} u^{j}(T-t, x), \quad u^{j}(T-t, x):=E_{x}\left[\mathbb{1}_{O^{j}}\left(L_{T}\right) \mathrm{e}^{-\int_{t}^{T} r_{h} \mathrm{~d} h}\right] .
$$

Corollary 5.3 For $\epsilon>0$ and $\alpha \in(0,2]$, let L be a time-inhomogeneous Lévy process such that $E\left[\mathrm{e}^{\epsilon\left|L_{t}\right|}\right]<\infty$ for every $t \leq T$ and its symbol $A$ satisfies (A2)-(A4) for the index $\alpha$ and every weight $\eta \in \mathbb{R}^{d}$ with $|\eta|<\epsilon$. Then for every $0 \leq t<T$, the price of the zero-coupon bond in model (5.5) is given as

$$
P(t, T)=\sum_{j=1}^{2^{d}} u^{j}\left(T-t, L_{t}\right) \quad \text { a.s. }
$$

where $u^{j}$ is the unique weak solution in $W^{1}\left(0, T ; H_{\eta^{j}}^{\alpha / 2}\left(\mathbb{R}^{d}\right), L_{\eta^{j}}^{2}\left(\mathbb{R}^{d}\right)\right)$ of

$$
\dot{u}^{j}+\mathcal{A}_{T-t} u^{j}+r u^{j}=0, \quad u^{j}(0)=\mathbb{1}_{O^{j}} .
$$

Proof The assumptions yield that for each $j=1, \ldots, 2^{d}$, conditions (A1)-(A4) are satisfied for weight $\eta^{j}$ and index $\alpha$. According to Remark 4.1, the distribution of $L_{T}$ has a Lebesgue density, which yields (5.9). Now the assertion follows directly from Theorem 3.4.

It is worth mentioning that with the same technique, we can characterize prices of options on a zero-coupon bond by solutions of PIDEs. A distinctive feature of the resulting PIDE is that the solution $u$ of (5.9) appears as the initial condition. Its initial condition thus is given by the solutions to the PIDEs (5.10).

Interesting related applications are bankruptcy probabilities in the model of [2], the value of barrier strategies in the bankruptcy model of [3], and reduced-form modeling of credit risk as in [30]. 


\subsection{Laplace transform of occupation times}

We characterize Laplace transforms of occupation times of time-inhomogeneous Lévy processes via weak solutions of PIDEs. Setting $\kappa:=\mathbb{1}_{D}$ for $D \subset \mathbb{R}^{d}$ Borelmeasurable, $f:=0$, initial condition $g:=1$, and inserting $L_{0}=x$, Eq. (3.8) from Theorem 3.4 becomes

$$
u(T, x)=E_{x}\left[\mathrm{e}^{-\gamma \int_{0}^{T} \mathbb{1}_{D}\left(L_{h}\right) \mathrm{d} h}\right]
$$

which is the Laplace transform at $\gamma$ of the occupation time $\int_{0}^{T} \mathbb{1}_{D}\left(L_{h}\right) \mathrm{d} h$ that the process $L$ spends in the domain $D$ until time $T$. Landriault et al. [32] analyze Laplace transforms of occupation times of spectrally negative Lévy processes using fluctuation identities. We characterize these transforms for a wide class of timeinhomogeneous Lévy processes by parabolic PIDEs. Let us point out that the assertion is not restricted to spectrally negative processes as the examples of NIG and tempered stable processes show; see Sect. 4.

Splitting the corresponding initial condition according to Remark 5.2, let

$$
u^{j}(T, x):=E_{x}\left[\mathbb{1}_{O^{j}}\left(L_{T}\right) \mathrm{e}^{-\gamma \int_{0}^{T} \mathbb{1}_{D}\left(L_{h}\right) \mathrm{d} h}\right] .
$$

Arguing as in the proof of Corollary 5.3 and applying Corollary 3.5, we obtain

Corollary 5.4 For $\epsilon>0$ and $\alpha \in(0,2]$, let L be a time-inhomogeneous Lévy process such that $E\left[\mathrm{e}^{\epsilon\left|L_{t}\right|}\right]<\infty$ for every $t \leq T$ and its symbol $A$ satisfies (A2)-(A4) for the index $\alpha$ and every weight $\eta \in \mathbb{R}^{d}$ with $|\eta|<\epsilon$. Let $\eta^{j}:=-\epsilon d^{-1 / 2} p^{j}$ as in (5.8). Then $u^{j}$ from (5.12) is the unique weak solution in $W^{1}\left(0, T ; H_{\eta^{j}}^{\alpha / 2}\left(\mathbb{R}^{d}\right), L_{\eta^{j}}^{2}\left(\mathbb{R}^{d}\right)\right)$ of

$$
\dot{u}^{j}+\mathcal{A}_{T-t} u^{j}+\mathbb{1}_{D} u^{j}=0, \quad u^{j}(0)=\mathbb{1}_{O^{j}},
$$

and $u$ from (5.11) is given by

$$
u(T, x)=\sum_{j=1}^{2^{d}} u^{j}(T, x) .
$$

If $d=1$ and $\alpha>1$, then $x \mapsto u(t, x):=E_{x}\left[\mathrm{e}^{-\gamma \int_{0}^{t} \mathbb{1}_{D}\left(L_{h}\right) \mathrm{d} h}\right]$ is $\lambda$-Hölder-continuous with $\lambda=\frac{\alpha-1}{2}$ for each $t \in[0, T]$ and in particular also is continuous.

\subsection{Penalization of the domain}

Observe that the limit of $\mathrm{e}^{-\gamma \int_{0}^{T} \mathbb{1}_{\bar{D}^{c}}\left(L_{h}\right) \mathrm{d} h}$ as $\gamma \rightarrow \infty$ links occupation times to exit times. This idea lies at the basis of the repeated use of occupation times for modeling. Moreover, it opens a way to establish a Feynman-Kac-type representation like (3.8) for boundary value problems. In the language of diffusions, a particle present in the outer domain is penalized more and more strongly until it is finally killed the moment it leaves the domain. For jump-diffusion processes, the argument is outlined 
in [5]. In [17], a similar technique is used for time-inhomogeneous Lévy processes. Interestingly for finance, the resulting Feynman-Kac-type representation serves to characterize prices of barrier and lookback options in pure jump models. The argument is based on the following result and the convergence of solutions for a sequence of killing rates of indicator-type given by $\kappa^{\lambda}(x):=\lambda \mathbb{1}_{\bar{D}^{c}}(x)$ for $\lambda \rightarrow \infty$.

Corollary 5.5 For $\alpha \in(0,2]$ and $\eta \in \mathbb{R}^{d}$, let $L$ be a time-inhomogeneous Lévy process satisfying assumptions (A1)-(A4). Let $f \in L^{2}\left(0, T ; H_{\eta}^{\ell}\left(\mathbb{R}^{d}\right)\right)$ for some $\ell \geq 0$ with $\ell>(d-\alpha) / 2, g \in L_{\eta}^{2}\left(\mathbb{R}^{d}\right), \kappa:[0, T] \times \mathbb{R}^{d} \rightarrow \mathbb{R}$ be measurable and bounded, $\lambda>0$ and $D \subset \mathbb{R}^{d}$ open. Then the unique weak solution $u^{\lambda}$ in the space $W^{1}\left(0, T ; H_{\eta}^{\alpha / 2}\left(\mathbb{R}^{d}\right), L_{\eta}^{2}\left(\mathbb{R}^{d}\right)\right)$ of

$$
\partial_{t} u^{\lambda}+\mathcal{A}_{T-t} u^{\lambda}+\kappa_{T-t} u^{\lambda}+\lambda \mathbb{1}_{\bar{D}} u^{\lambda}=f, \quad u^{\lambda}(0)=g,
$$

has for every $t \in(0, T]$ almost surely the stochastic representation

$$
\begin{aligned}
u^{\lambda}\left(T-t, L_{t}\right)=E[ & g\left(L_{T}\right) \mathrm{e}^{-\int_{t}^{T} \kappa_{h}\left(L_{h}\right) \mathrm{d} h} \mathrm{e}^{-\lambda \int_{t}^{T} \mathbb{1}_{\bar{D}^{c}\left(L_{h}\right) \mathrm{d} h}} \\
& \quad+\int_{t}^{T} f\left(T-s, L_{s}\right) \mathrm{e}^{-\int_{t}^{s} \kappa_{h}\left(L_{h}\right) \mathrm{d} h} \mathrm{e}^{\left.-\lambda \int_{t}^{s} \mathbb{1}_{\bar{D}^{c}\left(L_{h}\right) \mathrm{d} h} \mathrm{~d} s \mid \mathcal{F}_{t}\right] .}
\end{aligned}
$$

Proof The assertion follows directly from Theorem 3.4.

\section{Numerical implementation}

Let us now explore the practical benefits of our Feynman-Kac-type result. We therefore implement a numerical scheme to solve the Kolmogorov equation (1.1) for pricing path-dependent options in jump models. We specify a class of employee options so as to shed light on the effect of a discontinuous killing rate. In order to give insight into the technique, we choose as numerical scheme the wavelet Galerkin method as developed by [33] for European option pricing. This is a very powerful method which uses compression techniques and can be adapted to more involved pricing problems, as we demonstrate by incorporating killing rates. The implementation requires some results from the classical theory on numerical analysis of partial differential equations. In addition, the jump part of the operator needs some special treatment. We take care of the derivation of the discrete scheme by presenting the discretization steps (1)-(6) below.

We specify a type of employee options as described in Sect. 5.1. So as to include penalizations of low stock values permanently rather than only at a fixed maturity, we combine a call option with an indicator-type killing rate. We specify the latter as instantaneous penalization for stock values below a fixed level by setting $\kappa(S):=-\lambda \mathbb{1}_{(-\infty, B]}(S)$ with a scale factor $\lambda>0$ and level $B$ in $(5.1)$, i.e., the payout at maturity is given by

$$
G\left(S_{T}\right) \mathrm{e}^{-\int_{0}^{T} \lambda \mathbb{1}_{(-\infty, B]}\left(S_{h}\right) \mathrm{d} h},
$$


where $G(S):=\max \{S-K, 0\}$. As driving process $L$ in the model $S=S_{0} \mathrm{e}^{L}$, we choose a pure jump Lévy process from the family of CGMY processes described in Example 4.9 with parameters $C>0, G>1, M>0, Y \in[1,2)$, and whose drift $b$ is given by the no-arbitrage condition (5.3). Then, according to Example 4.9, the process and its symbol satisfy conditions (A1)-(A4), with weight $\eta \in(-G,-1)$ and index $\alpha=Y$.

We now fix a weight $\eta \in(-G,-1)$, denote by $\mathcal{A}$ the Kolmogorov operator of the process and let $\widetilde{G}(x):=G\left(\mathrm{e}^{x}\right)$ and $\tilde{\kappa}(x):=-\kappa\left(\mathrm{e}^{x}\right)$. According to Corollary 5.1, we obtain the fair price of the option by computing the unique weak solution $u \in W^{1}\left(0, T ; H_{\eta}^{Y / 2}(\mathbb{R}), L_{\eta}^{2}(\mathbb{R})\right)$ of

$$
\dot{u}+\mathcal{A} u+(r+\tilde{\kappa}) u=0, \quad u(0)=\widetilde{G} .
$$

In order to prepare the discretization with finite elements, we first modify and then localize the equation to a bounded interval. The variational formulation of the resulting equation then allows us to discretize the space with a Galerkin method. Finally, the time discretization completes the fully discrete scheme. In more detail, we proceed along the following steps:

1. (Modification of the equation) We first choose a function $\psi$ in the space $W^{1}\left(0, T ; H_{\eta}^{Y / 2}(\mathbb{R}), L_{\eta}^{2}(\mathbb{R})\right)$ such that the difference $\phi:=u-\psi$ lies in the space $W^{1}\left(0, T ; H^{Y / 2}(\mathbb{R}), L^{2}(\mathbb{R})\right)$ and $|\phi(t, x)| \rightarrow 0$ for $|x| \rightarrow \infty$. Then $\phi$ is the unique weak solution of the modified equation

$$
\dot{\phi}+\mathcal{A} \phi+(r+\tilde{\kappa}) \phi=f, \quad \phi(0)=\widetilde{G}-\psi(0) .
$$

2. (Truncation to a bounded domain) We localize the equation to a bounded interval $\left(R_{1}, R_{2}\right)$ with zero constraints outside of the interval. Here, we encounter for the first time a conceptual difference between jump and non-jump processes: The jump part of the process renders the operator $\mathcal{A}$ nonlocal. It therefore does not suffice to specify zero boundary conditions. Rather, the values have to be set on the whole outer domain $\mathbb{R} \backslash\left(R_{1}, R_{2}\right)$. Formally, we incorporate these zero constraints by setting $\widetilde{L}^{2}\left(R_{1}, R_{2}\right):=\left\{g \in L^{2}(\mathbb{R})|g|_{\left[R_{1}, R_{2}\right]^{c}}=0\right\}$ and defining the solution space as

$$
\widetilde{H}^{Y / 2}\left(R_{1}, R_{2}\right):=\left\{u \in H^{Y / 2}(\mathbb{R})|u|_{\left[R_{1}, R_{2}\right]^{c}}=0\right\} .
$$

More precisely, instead of solving (6.2), we approximate the unique weak solution $\tilde{\phi} \in W^{1}\left(0, T ; \widetilde{H}^{Y / 2}\left(R_{1}, R_{2}\right), \widetilde{L}^{2}\left(R_{1}, R_{2}\right)\right)$ of

$$
\dot{\tilde{\phi}}+\mathcal{A} \tilde{\phi}+(r+\tilde{\kappa}) \tilde{\phi}=f, \quad \tilde{\phi}(0)=(\widetilde{G}-\psi(0)) \mathbb{1}_{\left(R_{1}, R_{2}\right)} .
$$

We now have to realize that we have changed the problem and that we need to control the resulting error $\|\phi-\tilde{\phi}\|$ with an appropriate norm $\|\cdot\|$. Put differently, we have to choose the function $\psi$ in step 1 in such a way that the error $\|\phi-\tilde{\phi}\|$ decays fast as $-R_{1}, R_{2} \rightarrow \infty$. 
3. (Variational formulation of the equation) The weak solution $\tilde{\phi}$ in the space $W^{1}\left(0, T ; \widetilde{H}^{Y / 2}\left(R_{1}, R_{2}\right), \widetilde{L}^{2}\left(R_{1}, R_{2}\right)\right)$ solves the operator equation (6.3) if and only if $\tilde{\phi}$ satisfies the initial condition of (6.3) as a limit in $\widetilde{L}^{2}$, that is,

$$
\lim _{t \rightarrow 0} \tilde{\phi}(t)=\left(\widetilde{G}-\psi(0) \mathbb{1}_{\left(R_{1}, R_{2}\right)}\right) \quad \text { in } \widetilde{L}^{2}\left(R_{1}, R_{2}\right),
$$

and for all $v \in C_{0}^{\infty}(0, T)$ and $\varphi \in \widetilde{H}^{Y / 2}\left(R_{1}, R_{2}\right)$,

$$
-\int_{0}^{T}\langle\tilde{\phi}(t), \varphi\rangle_{L^{2}} \dot{v}(t) \mathrm{d} t+\int_{0}^{T} a(\tilde{\phi}(t), \varphi) v(t) \mathrm{d} t=\bar{f}(\varphi, v)
$$

with the bilinear form $a: \widetilde{H}^{Y / 2}\left(R_{1}, R_{2}\right) \times \widetilde{H}^{Y / 2}\left(R_{1}, R_{2}\right) \rightarrow \mathbb{R}$ corresponding to the operator $\mathcal{A}$ according to (2.3) and $\bar{f}(\varphi, v):=\int_{0}^{T}\langle f(t), \varphi\rangle_{L^{2}} \nu(t) \mathrm{d} t$. For simplicity of presentation, we assume from now on that $\psi$ is constant in time.

4. (Space discretization with a Galerkin method) Coming to the heart of the Galerkin method, we choose a countable Riesz basis $\left\{w_{1}, w_{2}, \ldots\right\}$ of $\widetilde{H}^{Y / 2}\left(R_{1}, R_{2}\right)$ and define

$$
X_{n}:=\operatorname{span}\left\{w_{1}, \ldots, w_{n}\right\} \quad \text { for all } n \in \mathbb{N} .
$$

Since $\widetilde{H}^{Y / 2}\left(R_{1}, R_{2}\right)$ is dense in $\widetilde{L}^{2}\left(R_{1}, R_{2}\right)$, we may further choose $h_{n}$ in $X_{n}$ such that $h_{n} \rightarrow \tilde{\phi}(0)$ in $\widetilde{L}^{2}\left(R_{1}, R_{2}\right)$. We obtain the Galerkin equations for each fixed $n \in \mathbb{N}$ simply by restricting the variational equation (6.4). The resulting problem is: Find $a$ function $v_{n} \in W^{1}\left(0, T ; X_{n} ; \widetilde{L}^{2}\left(R_{1}, R_{2}\right) \cap X_{n}\right)$ that satisfies for all $\chi \in C_{0}^{\infty}(0, T)$ and $\varphi \in X_{n}$

$$
\begin{gathered}
-\int_{0}^{T}\left\langle v_{n}(t), \varphi\right\rangle_{L^{2}} \dot{\chi}(t) \mathrm{d} t+\int_{0}^{T} a\left(v_{n}(t), \varphi\right) \chi(t) \mathrm{d} t=\bar{f}(\varphi, \chi), \\
v_{n}(0)=h_{n} .
\end{gathered}
$$

Elegantly, the classical theory guarantees the convergence of the sequence $\left(v_{n}\right)$ to $\tilde{\phi}$ already in this abstract setting. For more details, we refer to [45, Theorem 23.A and Remark 23.25]. The actual performance of the scheme, however, critically depends on the choice of the Riesz basis, which determines the rate of convergence.

5. (Matrix formulation of (6.5)) Using the linearity of the operators, we can simplify (6.5). Namely, it is enough to insert the basis functions $w_{1}, \ldots, w_{n}$ as test functions $\varphi \in X_{n}$ in (6.5). Then, denoting $h_{n}:=\sum_{k=1}^{n} \alpha_{k} w_{k}$ and $v_{n}(t):=\sum_{k=1}^{n} V_{k}(t) w_{k}$, equation (6.5) turns out to be equivalent to

$$
\begin{aligned}
\sum_{k=1}^{n} \dot{V}_{k}(t)\left\langle w_{k}, w_{j}\right\rangle_{L^{2}}+\sum_{k=1}^{n} V_{k}(t) a\left(w_{k}, w_{j}\right) & =-a\left(\psi, w_{j}\right), \\
V_{k}(0) & =\alpha_{k} \quad \text { for all } k=1, \ldots, n .
\end{aligned}
$$

Written in matrix form, the problem is to find $V:[0, T] \rightarrow \mathbb{R}^{n}$ such that 


$$
\begin{aligned}
M \dot{V}(t)+A V(t) & =F, \\
V(0) & =\alpha,
\end{aligned}
$$

where $F=\left(F_{1}, \ldots, F_{n}\right)^{\top}$ with $F_{k}=-a\left(\psi, w_{k}\right)$ for every $k=1, \ldots, n$ and with $\alpha=\left(\alpha_{1}, \ldots, \alpha_{n}\right)^{\top}$, and the mass matrix $M$ and stiffness matrix $A$ are

$$
M_{j k}=\left\langle w_{k}, w_{j}\right\rangle_{L^{2}}, \quad A_{j k}=a\left(w_{k}, w_{j}\right) \quad \text { for all } j, k=1, \ldots, n .
$$

Let us mention two critical points that arise in our setting. First, approximation errors in the entries of the stiffness matrix $A$ typically lead to significant numerical errors of the resulting scheme. As a consequence, they have to be computed with high precision. Second, due to the nonlocal nature of the operator $\mathcal{A}$, the matrix $A$ is fully populated. This leads to a high computational cost of the solution scheme, which can be reduced considerably by using compression techniques.

6. (Time discretization) Having reached (6.6) and (6.7), we are finally left with solving a linear system of ordinary differential equations. A variety of discretization methods for these types of equations is available, for instance Euler schemes.

To illustrate the numerical effect of the killing rate, we use an implementation subject to the following specifications in the steps $1-6:{ }^{1}$

- The equation is modified according to $\psi(t, x):=\psi(x):=\max \left(\mathrm{e}^{x}-K, 0\right)$. Dominated convergence yields $|u(t, x)-\psi(x)| \rightarrow 0$ for $|x| \rightarrow \infty$. For a similar situation, Proposition 4.1 in [10] shows exponential convergence. We conjecture that also in our setting, we have an exponential decay of the difference $|\phi-\tilde{\phi}|$.

- As Riesz basis, a wavelet basis of first polynomial order is chosen and combined with a compression technique replacing the stiffness matrix by a sparse one. We refer to Sect. 12.2.2 in the monograph of Hilber et al. [23] for a presentation of the wavelet compression technique and to [36] for a related error analysis.

- As time discretization, an $h p$-discontinuous Galerkin method is chosen, as the initial condition is not differentiable and a scheme selecting more time points at the beginning is advantageous. For details, we refer to Sect. 12.3 in [23].

In our numerical experiments, we consider different domains on which the killing rate is active. Each of these domains is specified by a location parameter $B$ according to $\tilde{\kappa}(x)=\lambda \mathbb{1}_{(-\infty, \log B)}(x)$. Figure 1 depicts our results for $B=K=100$ and $B=70$. In both cases, the maturity (in years) is set to $T=1$ and the strike to $K=100$. The parameters of the process are set to $C=0.01560, G=0.0767, M=7.55$ and $Y=1.2996$. Notice that the scales of the graphs on the left side are chosen differently from those on the right side.

We see that the killing rate of indicator-type displays an effect in all considered cases. While the difference between the call and the employee options peaks around the level $S_{0}=B$, the killing rate affects prices globally with fast decay on both sides. The effects are stronger for higher scale parameters $\lambda$. This leads to a monotone order of the price curves; the higher the scale parameter $\lambda$, the lower the price when all other parameters are kept equal.

\footnotetext{
${ }^{1}$ The author gratefully acknowledges Christoph Schwab and his working group for letting her use their code, which implements the Galerkin method for pricing European call options in CGMY models.
} 

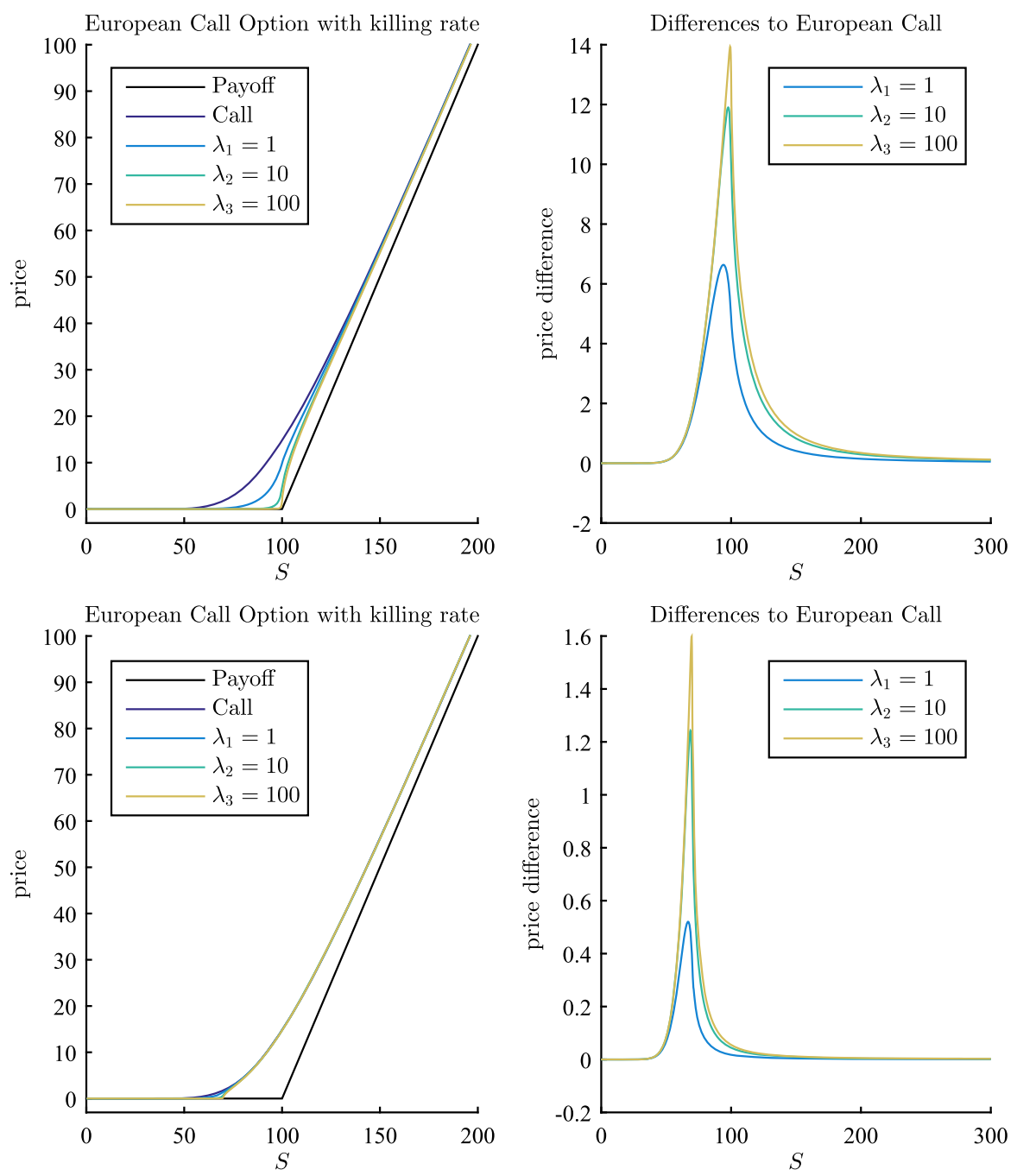

Fig. 1 Effect of different killing rates on the prices of employee options as defined by (6.1). The call option price is the special case of a zero killing rate. (Top) $B=K=100$. (Bottom) $B=70$. (Left) Payout of the call option along with the prices of the call option as well as of the employee options with $\lambda=1,10,100$. (Right) Differences between the call price (without killing rate) and the prices of the employee options (with different positive killing rates)

\section{Robustness of the weak solutions}

We provide a robustness result that shows that small perturbations of the data $f$ and $g$ and, more critically, of the bilinear form $a$ only have a small effect on the weak solution of the Kolmogorov equation (1.1). The result is crucial for the limit procedure in our derivation of the Feynman-Kac-type representation in Theorem 3.4. 
Let $X \hookrightarrow H \hookrightarrow X^{*}$ be a Gelfand triplet. For $t \in[0, T]$ and each $n \in \mathbb{N}$, let $\mathcal{A}_{t}^{n}$ (respectively $\mathcal{A}_{t}$ ) be an operator with associated real-valued bilinear form $a_{t}^{n}$ (respectively $a_{t}$ ). We introduce the following set of conditions:

(An1) There exists a constant $C_{1}>0$ such that uniformly for all $n \in \mathbb{N}, t \in[0, T]$ and $u, v \in X$,

$$
\max \left\{\left|a_{t}^{n}(u, v)\right|,\left|a_{t}(u, v)\right|\right\} \leq C_{1}\|u\|_{X}\|v\|_{X} .
$$

(An2) There exist constants $C_{2}, C_{3}>0$ such that uniformly for all $n \in \mathbb{N}, t \in[0, T]$ and $u \in X$,

$$
\min \left\{a_{t}^{n}(u, u), a_{t}(u, u)\right\} \geq C_{2}\|u\|_{X}^{2}-C_{3}\|u\|_{H}^{2} .
$$

(An3) There exists a sequence of functionals $F_{n}: L^{2}(0, T ; H) \rightarrow \mathbb{R}_{+}$such that for all $n \in \mathbb{N}$ and $u, v \in L^{2}(0, T ; H)$, both $F_{n}(u) \rightarrow 0$ for $n \rightarrow \infty$ and

$$
\int_{0}^{T}\left|\left(a_{t}^{n}-a_{t}\right)(u(t), v(t))\right| \mathrm{d} t \leq F_{n}(u)\|v\|_{L^{2}(0, T ; H)} .
$$

Lemma 7.1 Let the operators $\mathcal{A}$ and $\mathcal{A}^{n}$ for $n \in \mathbb{N}$ satisfy(An1)-(An3). Suppose that $f^{n}, f \in L^{2}(0, T ; H)$ satisfy $f^{n} \rightarrow f$ in $L^{2}\left(0, T ; X^{*}\right)$ and $g^{n}, g \in H$ satisfy $g^{n} \rightarrow g$ in $H$. Then the sequence of unique weak solutions $u^{n} \in W^{1}(0, T ; Y, H)$ of

$$
\dot{u}^{n}+\mathcal{A}_{t}^{n} u^{n}=f^{n}, \quad u^{n}(0)=g^{n}
$$

converges strongly in $L^{2}(0, T ; X) \cap C(0, T ; H)$ to the unique weak solution $u$ in the space $W^{1}(0, T ; X, H)$ of

$$
\dot{u}+\mathcal{A}_{t} u=f, \quad u(0)=g .
$$

Proof Fix $n \in \mathbb{N}$ and let $u^{n}, u \in W^{1}(0, T ; X, H)$ be the unique weak solutions of (7.3) and (7.4) and $w^{n}:=u-u^{n}$. Subtracting (7.3) from (7.4) and inserting $w^{n}$ as test function yields for every $t \in[0, T]$

$$
\begin{aligned}
\int_{0}^{t}\left(\dot{w}^{n}(s), w^{n}(s)\right) \mathrm{d} s+\int_{0}^{t} a_{s}^{n}\left(w^{n}(s), w^{n}(s)\right) \mathrm{d} s \\
\quad=\int_{0}^{t}\left(f^{n}(s)-f(s), w^{n}(s)\right) \mathrm{d} s+\int_{0}^{t}\left(a_{s}^{n}-a_{s}\right)\left(u(s), w^{n}(s)\right) \mathrm{d} s .
\end{aligned}
$$

We insert $\int_{0}^{t}\left(\dot{w}^{n}(s), w^{n}(s)\right) \mathrm{d} s=\frac{1}{2}\left(\left\|w^{n}(t)\right\|_{H}^{2}-\left\|w^{n}(0)\right\|_{H}^{2}\right)$. Using (7.1), (7.2) and the inequality of Young and subsequently applying the lemma of Gronwall yields the existence of constants $c_{1}, c_{2}>0$ such that

$$
\begin{aligned}
& \sup _{t \in[0, T]}\left\|w^{n}(t)\right\|_{H}^{2}+c_{1}\left\|w^{n}\right\|_{L^{2}(0, T ; X)}^{2} \\
& \quad \leq c_{2}\left(\left|F_{n}(u)\right|^{2}+\left\|f^{n}-f\right\|_{L^{2}\left(0, T ; X^{*}\right)}^{2}+\left\|g^{n}-g\right\|_{H}^{2}\right)
\end{aligned}
$$

with $F_{n}$ from condition (An3). Hence $u^{n} \rightarrow u$ converges strongly in $L^{2}(0, T ; X)$ and in $C(0, T ; H)$, which proves the lemma. 


\section{Proof of the Feynman-Kac-type formula, part (ii) of Theorem 3.4}

The key steps in the proof of the Feynman-Kac-type formula in Theorem 3.4 are first applying Itô's formula with the help of the regularity assertion in Lemma 8.1 below, second invoking the convergence of regularized solutions to the solution of the Kolmogorov equation (1.1) due to the robustness result in Lemma 7.1, and third linking convergence in $L_{\eta}^{2}\left(\mathbb{R}^{d}\right)$ (respectively $L^{2}\left(0, T ; H_{\eta}^{l}\left(\mathbb{R}^{d}\right)\right)$ ) to the convergence of conditional expectations via Lemma 8.2.

Lemma 8.1 For $\eta \in \mathbb{R}^{d}$ and $\alpha>0$, let $\mathcal{A}$ be a pseudo-differential operator whose symbol $A$ has Sobolev index $\alpha$ uniformly in $[0, T] \times R_{\eta}$, and suppose that the mapping $t \mapsto A_{t}(\xi-i \eta)$ is continuous for every $\xi \in \mathbb{R}^{d}$. For $\kappa \in L^{\infty}\left([0, T] \times \mathbb{R}^{d}\right)$, $g \in L_{\eta}^{2}\left(\mathbb{R}^{d}\right)$ and $f \in L^{2}\left(0, T ; H_{\eta}^{\alpha / 2}\left(\mathbb{R}^{d}\right)\right)$, let $u \in W^{1}\left(0, T ; H_{\eta}^{\alpha / 2}\left(\mathbb{R}^{d}\right), L_{\eta}^{2}\left(\mathbb{R}^{d}\right)\right)$ be the unique weak solution of

$$
\begin{aligned}
\dot{u}+\mathcal{A}_{T-t} u+\kappa_{T-t} u & =f, \\
u(0) & =g .
\end{aligned}
$$

Then the following assertions hold:

(i) Let $m \geq 1$. Assume $g \in H_{\eta}^{(m-1) \alpha / 2}\left(\mathbb{R}^{d}\right), f \in L^{2}\left(0, T ; H_{\eta}^{(m-1) \alpha / 2}\left(\mathbb{R}^{d}\right)\right)$ and $\kappa h \in L^{2}\left(0, T ; H_{\eta}^{k \alpha / 2}\left(\mathbb{R}^{d}\right)\right)$ for all $1 \leq k \leq m$ and $h \in L^{2}\left(0, T ; H_{\eta}^{k \alpha / 2}\left(\mathbb{R}^{d}\right)\right)$. Then $u \in L^{2}\left(0, T ; H_{\eta}^{m \alpha / 2}\left(\mathbb{R}^{d}\right)\right)$ and $\dot{u} \in L^{2}\left(0, T ; H_{\eta}^{(m-2) \alpha / 2}\left(\mathbb{R}^{d}\right)\right)$.

(ii) Assume $g \in H_{\eta}^{\beta}\left(\mathbb{R}^{d}\right)$ for $\beta=m+d / 2+\max (\alpha, 1 / 2), f \in L^{2}\left(0, T ; H_{\eta}^{\gamma}\left(\mathbb{R}^{d}\right)\right)$ for $\gamma=m+(d+1) / 2$ and $\kappa \in C_{0}^{\infty}\left([0, T] \times \mathbb{R}^{d}\right)$. Then for every multi-index $k=\left(k_{1}, \ldots, k_{d}\right)$ with $|k| \leq m$, the derivative $\left(1+\partial_{t}\right) D^{k} u$ lies in the space $C\left([0, T] \times \mathbb{R}^{d}\right)$. If, moreover, $\mathcal{A}$ is the Kolmogorov operator of a Lévy process and $f$ is continuous, then (8.1) holds pointwise for all $(t, x) \in(0, T] \times \mathbb{R}^{d}$.

Proof We derive the regularity assertion by explicit operations on the Fourier transform of the unique weak solution $u \in W^{1}\left(0, T ; H_{\eta}^{\alpha / 2}\left(\mathbb{R}^{d}\right), L_{\eta}^{2}\left(\mathbb{R}^{d}\right)\right)$ of (8.1) and (8.2). We show the identity

$$
u=\tilde{u}:=u^{1}+u^{2}+u^{3}
$$

with

$$
\begin{aligned}
& \mathcal{F}_{\eta}\left(u^{1}(t)\right):=\mathcal{F}_{\eta}(g) \mathrm{e}^{-\int_{T-t}^{T} A_{u}(\cdot-i \eta) \mathrm{d} u}, \\
& \mathcal{F}_{\eta}\left(u^{2}(t)\right):=\int_{0}^{t} \mathcal{F}_{\eta}(f(s)) \mathrm{e}^{-\int_{T-t}^{T-s} A_{u}(\cdot-i \eta) \mathrm{d} u} \mathrm{~d} s, \\
& \mathcal{F}_{\eta}\left(u^{3}(t)\right):=-\int_{0}^{t} \mathcal{F}_{\eta}(\kappa u(s)) \mathrm{e}^{-\int_{T-t}^{T-s} A_{\lambda}(\cdot-i \eta) \mathrm{d} \lambda} \mathrm{d} s
\end{aligned}
$$

and hence

$$
\partial_{t} \mathcal{F}_{\eta}\left(u^{1}(t)\right)=-A_{T-t}(\cdot-i \eta) \mathcal{F}_{\eta}\left(u^{1}(t)\right),
$$




$$
\begin{aligned}
& \partial_{t} \mathcal{F}_{\eta}\left(u^{2}(t)\right)=-A_{T-t}(\cdot-i \eta) \mathcal{F}_{\eta}\left(u^{2}(t)\right)+\mathcal{F}_{\eta}(f(t)), \\
& \partial_{t} \mathcal{F}_{\eta}\left(u^{3}(t)\right)=-A_{T-t}(\cdot-i \eta) \mathcal{F}_{\eta}\left(u^{3}(t)\right)-\mathcal{F}_{\eta}(\kappa u(t)) .
\end{aligned}
$$

In particular, $\tilde{u}$ satisfies (8.1). The inequality (4.1) from Remark 4.1 with constants $C_{1}, C_{2}>0$ and the inequality of Cauchy-Schwarz guarantee the existence of constants $c_{1}, c_{2}>0$ which are such that for all $(t, \xi) \in[0, T] \times \mathbb{R}^{d}$,

$$
\begin{aligned}
\left|\mathcal{F}_{\eta}\left(u^{1}(t)\right)(\xi)\right| & \leq C_{1}\left|\mathcal{F}_{\eta}(g)(\xi)\right| \mathrm{e}^{-t C_{2}|\xi|^{\alpha}} \\
\left|\mathcal{F}_{\eta}\left(u^{j}(t)\right)(\xi)\right| & \leq C_{1}\left(\int_{0}^{t}\left|\mathcal{F}_{\eta}(f(s))(\xi)\right|^{2} \mathrm{~d} s\right)^{1 / 2}\left(\int_{0}^{t} \mathrm{e}^{-(t-s) 2 C_{2}|\xi|^{\alpha}} \mathrm{d} s\right)^{1 / 2} \\
& \leq c_{2}\left(\int_{0}^{T}\left|\mathcal{F}_{\eta}\left(f^{j}(s)\right)(\xi)\right|^{2}(1+|\xi|)^{-\alpha} \mathrm{d} s\right)^{1 / 2}
\end{aligned}
$$

as well as

$$
\begin{aligned}
& \left|\mathcal{F}_{\eta}\left(\partial_{t} u^{1}(t)\right)(\xi)\right| \leq c_{1}\left|\mathcal{F}_{\eta}(g)(\xi)\right|(1+|\xi|)^{\alpha} \mathrm{e}^{-t C_{2}|\xi|^{\alpha}}, \\
& \left|\mathcal{F}_{\eta}\left(\partial_{t} u^{j}(t)\right)(\xi)\right| \leq c_{2}\left(\int_{0}^{T}\left|\mathcal{F}_{\eta}\left(f^{j}(s)\right)(\xi)\right|^{2}(1+|\xi|)^{\alpha} \mathrm{d} s\right)^{1 / 2}+\left|\mathcal{F}_{\eta}\left(f^{j}(s)\right)(\xi)\right|,
\end{aligned}
$$

for $j=1,2$ with $f^{1}=f$ and $f^{2}=-\kappa u$. Hence there is a constant $c_{3}>0$ with

$$
\begin{aligned}
& \|\tilde{u}\|_{L^{2}\left(0, T ; H_{\eta}^{m \alpha / 2}\left(\mathbb{R}^{d}\right)\right)}+\left\|\partial_{t} \tilde{u}\right\|_{L^{2}\left(0, T ; H_{\eta}^{(m-2) \alpha / 2}\left(\mathbb{R}^{d}\right)\right)} \\
& \leq c_{3}\left(\|g\|_{H_{\eta}^{(m-1) \alpha / 2}\left(\mathbb{R}^{d}\right)}+\|f\|_{L^{2}\left(0, T ; H_{\eta}^{(m-1) \alpha / 2}\left(\mathbb{R}^{d}\right)\right)}+\|\kappa u\|_{L^{2}\left(0, T ; H_{\eta}^{(m-1) \alpha / 2}\left(\mathbb{R}^{d}\right)\right)}\right) .
\end{aligned}
$$

For $m=1$, by inserting $u \in L^{2}\left(0, T ; H_{\eta}^{\alpha / 2}\left(\mathbb{R}^{d}\right)\right)$ and $\kappa u \in L^{2}\left(0, T ; H_{\eta}^{\alpha / 2}\left(\mathbb{R}^{d}\right)\right)$, we obtain $\tilde{u} \in L^{2}\left(0, T ; H_{\eta}^{\alpha / 2}\left(\mathbb{R}^{d}\right)\right)$ and $\partial_{t} \tilde{u} \in L^{2}\left(0, T ; H_{\eta}^{-\alpha / 2}\left(\mathbb{R}^{d}\right)\right)$. In particular, $\tilde{u} \in W^{1}\left(0, T ; H_{\eta}^{\alpha / 2}\left(\mathbb{R}^{d}\right), L_{\eta}^{2}\left(\mathbb{R}^{d}\right)\right)$ is the unique weak solution $\tilde{u}=u$ of $(8.1)$ and (8.2). For $m=2$, it is thus sufficient to notice that $\kappa u \in L^{2}\left(0, T ; H_{\eta}^{\alpha / 2}\left(\mathbb{R}^{d}\right)\right)$ implies $u \in L^{2}\left(0, T ; H_{\eta}^{\alpha / 2}\left(\mathbb{R}^{d}\right)\right)$ and $\partial_{t} \tilde{u} \in L^{2}\left(0, T ; L_{\eta}^{2}\left(\mathbb{R}^{d}\right)\right)$. An iterative argument then yields part (i).

(ii) By the inequality of Cauchy-Schwarz and $\int_{\mathbb{R}^{d}}(1+|\xi|)^{-d-\epsilon} \mathrm{d} \xi<\infty$ if $\epsilon>0$, we obtain that for $\beta=m+d / 2+\max (\alpha, 1 / 2)$ and $\gamma=m+(d+1) / 2$, there exists a constant $c_{4}>0$ such that

$$
\begin{aligned}
& \int_{\mathbb{R}^{d}}\left|\left(1+\partial_{t}\right) \mathcal{F}_{\eta}\left(\left(u^{1}+u^{2}\right)(t)\right)(\xi)\right|(1+|\xi|)^{m} \mathrm{~d} \xi \\
& \leq c_{4}\left(\|g\|_{H_{\eta}^{\beta}\left(\mathbb{R}^{d}\right)}+\|f\|_{L^{2}\left(0, T ; H_{\eta}^{\gamma}\left(\mathbb{R}^{d}\right)\right)}\right)<\infty .
\end{aligned}
$$

Moreover, both $t \mapsto \mathcal{F}_{\eta}(\tilde{u}(t))(\xi)$ and $t \mapsto \partial_{t} \mathcal{F}_{\eta}(\tilde{u}(t))(\xi)$ are continuous for each fixed $\xi \in \mathbb{R}^{d}$. Dominated convergence thus implies that we have 
$D_{x}^{k}\left(1+\partial_{t}\right)\left(u^{1}+u^{2}\right) \in C\left([0, T] \times \mathbb{R}^{d}\right)$ for every multi-index $k=\left(k_{1}, \ldots, k_{d}\right)$ with $|k| \geq 0$. Furthermore, there exists a constant $c_{5}>0$ such that

$$
\int_{\mathbb{R}^{d}}\left|\left(1+\partial_{t}\right) \mathcal{F}_{\eta}\left(u^{3}(t)\right)(\xi)\right|(1+|\xi|)^{m} \mathrm{~d} \xi \leq c_{5}\|\kappa u\|_{L^{2}\left(0, T ; H_{\eta}^{\gamma}\left(\mathbb{R}^{d}\right)\right)}<\infty .
$$

Dominated convergence yields $D_{x}^{k}\left(1+\partial_{t}\right) u^{3} \in C\left([0, T] \times \mathbb{R}^{d}\right)$ for every multi-index $k=\left(k_{1}, \ldots, k_{d}\right)$ with $|k| \geq 0$.

Now let $\mathcal{A}$ be the Kolmogorov operator of a time-inhomogeneous Lévy process. To establish (8.1) pointwise, we fix some $t \in(0, T)$ for which the equation holds (as operator equation). Then we choose a sequence $\left(u_{n}\right)$ in $C_{0}^{\infty}\left((0, T) \times \mathbb{R}^{d}\right)$ such that $u_{n}(t) \rightarrow u(t)$ in the norm of $H_{\eta}^{\alpha / 2}\left(\mathbb{R}^{d}\right)$ and $\dot{u}_{n}(t) \rightarrow \dot{u}$ in the norm of $L_{\eta}^{2}\left(\mathbb{R}^{d}\right)$. Moreover, let $\varphi \in C_{0}^{\infty}\left(\mathbb{R}^{d}\right)$. We notice that $\mathcal{A}_{T-t} u(t)$ is defined pointwise since $u(t) \in C^{2}\left(\mathbb{R}^{d}\right)$. An elementary manipulation and the continuity of the scalar product yield

$$
\int_{\mathbb{R}^{d}} \mathcal{A}_{T-t} u(t, x) \varphi(x) \mathrm{e}^{-2\langle\eta, x\rangle} \mathrm{d} x=\left\langle u(t), \mathcal{A}_{T-t}^{-\eta, *} \varphi\right\rangle_{L_{\eta}^{2}}=\lim _{n \rightarrow \infty}\left\langle u_{n}(t), \mathcal{A}_{T-t}^{-\eta, *} \varphi\right\rangle_{L_{\eta}^{2}}
$$

with the adjoint operator $\mathcal{A}_{T-t}^{-\eta, *}$ defined before Lemma A.2 below. Equation (A.1) from Appendix A and the continuity of the bilinear form imply

$$
\lim _{n \rightarrow \infty}\left\langle u_{n}(t), \mathcal{A}_{T-t}^{-\eta, *} \varphi\right\rangle_{L_{\eta}^{2}}=\lim _{n \rightarrow \infty} a_{T-t}\left(u_{n}(t), \varphi\right)=a_{T-t}(u(t), \varphi)
$$

and hence

$$
\langle\dot{u}(t), \varphi\rangle_{L_{\eta}^{2}}+\left\langle\mathcal{A}_{T-t} u(t), \varphi\right\rangle_{L_{\eta}^{2}}=\langle f(t), \varphi\rangle_{L_{\eta}^{2}} \quad \text { for all } \varphi \in C_{0}^{\infty}\left(\mathbb{R}^{d}\right) .
$$

The fundamental lemma of variational calculus yields that for a.e. $x \in \mathbb{R}^{d}$, we have $\dot{u}(t, x)+\mathcal{A}_{T-t} u(t, x)=f(t, x)$. Since we can choose $t$ arbitrarily from a dense subset in $(0, T)$, the assertion follows by continuity of $\dot{u}+\mathcal{A}_{T-} u-f$.

Lemma 8.2 For $\eta \in \mathbb{R}^{d}$ and $\alpha \in(0,2]$, let L be a time-inhomogeneous Lévy process with symbol $A=\left(A_{t}\right)_{t \in[0, T]}$ that satisfies the exponential moment condition $(A 1)$ and the Gårding condition (A3). Then:

(i) For every $t>0$, there exists a constant $C(t)>0$ such that uniformly for all $\varphi \in L_{\eta}^{2}\left(\mathbb{R}^{d}\right)$ and all $s \in[t, T]$,

$$
E\left[\left|\varphi\left(L_{S}\right)\right|\right] \leq C(t)\|\varphi\|_{L_{\eta}^{2}\left(\mathbb{R}^{d}\right)} .
$$

(ii) For $\ell>(d-\alpha) / 2$ and every $0 \leq t<T$, there exists a constant $C_{1}>0$ such that uniformly for all $\varphi \in L^{2}\left(0, T ; H_{\eta}^{\ell}\left(\mathbb{R}^{d}\right)\right)$,

$$
\left|E\left[\int_{t}^{T} \varphi\left(s, L_{s}\right) \mathrm{d} s \mid \mathcal{F}_{t}\right]\right| \leq C_{1}\|\varphi\|_{L^{2}\left(t, T ; H_{\eta}^{\ell}\left(\mathbb{R}^{d}\right)\right)}
$$


Proof (i) By Remark 4.1 and condition (A3), the distribution of $L_{t}$ has a Lebesgue density. Applying Parseval's identity, we obtain

$$
E\left[\left|\varphi\left(L_{t}\right)\right|\right]=\frac{1}{(2 \pi)^{d}} \int_{\mathbb{R}^{d}} \mathcal{F}(|\varphi|)(\xi-i \eta) \overline{\mathrm{e}^{-\int_{0}^{t} A_{s}(\xi-i \eta) \mathrm{d} s}} \mathrm{~d} \xi .
$$

Inserting (4.1) and the inequality of Cauchy-Schwarz then yields (i).

(ii) Without loss of generality, we can assume that $\varphi \geq 0$. Then we can write $E\left[\int_{t}^{T} \varphi\left(s, L_{s}\right) \mathrm{d} s \mid \mathcal{F}_{t}\right]=G\left(L_{t}\right)$ with

$$
G(y)=E\left[\int_{0}^{T-t} \varphi\left(s+t, L_{t+s}-L_{t}+y\right) \mathrm{d} s\right] .
$$

The theorem of Fubini and Parseval's identity imply

$$
G(y)=\frac{1}{(2 \pi)^{d}} \int_{\mathbb{R}^{d}} \int_{0}^{T-t} \mathcal{F}\left(\tau_{y} \varphi(s+t)\right)(\xi-i \eta) \overline{\mathrm{e}^{-\int_{0}^{s} A_{t+u}(\xi-i \eta) \mathrm{d} u}} \mathrm{~d} s \mathrm{~d} \xi,
$$

where $\tau_{y} f(x):=f(x+y)$. We notice that $\mathcal{F}_{\eta}\left(\tau_{y} f\right)(\xi)=\mathrm{e}^{-\langle\xi, y\rangle} \mathcal{F}_{\eta}(f)(\xi)$. Inserting the inequality of Cauchy-Schwarz and (4.1) with constants $C_{1}, C_{2}>0$, we see that for $\ell>d-\alpha$, there are constants $c_{1}, c_{2}>0$ such that

$$
\begin{aligned}
|G(y)| & \leq C_{1} \int_{\mathbb{R}^{d}}\left(\int_{0}^{T-t}\left|\mathcal{F}\left(\tau_{y} \varphi(s+t)\right)(\xi-i \eta)\right|^{2} \mathrm{~d} s \int_{0}^{T-t} \mathrm{e}^{-2 s C_{2}|\xi|^{\alpha}} \mathrm{d} s\right)^{1 / 2} \mathrm{~d} \xi \\
& \leq c_{1} \int_{0}^{T} \int_{\mathbb{R}^{d}}|\mathcal{F}(\varphi(s+t))(\xi-i \eta)|(1+|\xi|)^{\alpha} \mathrm{d} \xi \mathrm{d} s \\
& \leq c_{2}\|\varphi\|_{L^{2}\left(0, T ; H_{\eta}^{\ell / 2}\left(\mathbb{R}^{d}\right)\right)}
\end{aligned}
$$

This concludes the proof.

We are now in a position to prove part (ii) of Theorem 3.4.

Proof of Theorem 3.4, part (ii) First, assume that $t \mapsto A_{t}(\xi-i \eta)$ is continuous for all $\xi \in \mathbb{R}^{d}$. By density arguments and mollification, respectively, we can choose sequences $\left(g^{n}\right)$ in $C_{0}^{\infty}\left(\mathbb{R}^{d}\right),\left(f^{n}\right)$ in $C_{0}^{\infty}\left([0, T] \times \mathbb{R}^{d}\right)$ and $\left(\kappa^{n}\right)$ in $C_{0}^{\infty}\left([0, T] \times \mathbb{R}^{d}\right)$ such that for $n \rightarrow \infty$,

$$
\begin{array}{ll}
g^{n} \rightarrow g & \text { in } L_{\eta}^{2}\left(\mathbb{R}^{d}\right), \\
f^{n} \rightarrow f & \text { in } L^{2}\left(0, T ; H_{\eta}^{\ell}\left(\mathbb{R}^{d}\right)\right), \\
\kappa^{n} \rightarrow \kappa & \text { pointwise and } \sup _{n \in \mathbb{N}}\left\|\kappa^{n}\right\|_{L^{\infty}\left([0, T] \times \mathbb{R}^{d}\right)}<\infty .
\end{array}
$$

We denote, with a slight abuse of notation, by $a_{t}^{n}$ the bilinear form associated with $\mathcal{A}_{t}+\kappa_{t}^{n}$ and by $a_{t}$ the bilinear form associated with $\mathcal{A}_{t}+\kappa_{t}$. Then

$$
a_{t}^{n}(u, v)=a_{t}(u, v)+\left\langle\left(\kappa_{t}^{n}-\kappa_{t}\right) u, v\right\rangle_{L_{\eta}^{2}\left(\mathbb{R}^{d}\right)} \quad \text { for all } u, v \in H_{\eta}^{\alpha / 2}\left(\mathbb{R}^{d}\right) .
$$


Together with conditions (A1)-(A3) and the uniform boundedness of $\kappa^{n}$ and $\kappa$, we obtain the validity of conditions (An1) and (An2) from Sect. 6. Moreover, by (8.4) and the Cauchy-Schwarz inequality, we get

$$
\left|\int_{0}^{T}\left(a^{n}-a\right)(u(s), v(s)) \mathrm{d} s\right| \leq\left\|\left(\kappa^{n}-\kappa\right) u\right\|_{L^{2}\left(0, T ; L_{\eta}^{2}\left(\mathbb{R}^{d}\right)\right)}\|v\|_{L^{2}\left(0, T ; L_{\eta}^{2}\left(\mathbb{R}^{d}\right)\right)} .
$$

It follows from pointwise convergence of $\kappa^{n} \rightarrow \kappa$ and dominated convergence that for $n \rightarrow \infty$,

$$
F_{n}(u):=\left\|\left(\kappa^{n}-\kappa\right) u\right\|_{L^{2}\left(0, T ; L_{\eta}^{2}\left(\mathbb{R}^{d}\right)\right)} \longrightarrow 0 \quad \text { for all } u \in L^{2}\left(0, T ; L_{\eta}^{2}\left(\mathbb{R}^{d}\right)\right),
$$

and hence condition (An3) is satisfied. Let $u^{n} \in W^{1}\left(0, T ; H_{\eta}^{\alpha / 2}\left(\mathbb{R}^{d}\right), L_{\eta}^{2}\left(\mathbb{R}^{d}\right)\right)$ be the unique weak solution of

$$
\dot{u}^{n}+\mathcal{A}_{t} u^{n}+\kappa_{t}^{n} u^{n}=f^{n}, \quad u^{n}(0)=g^{n} .
$$

Lemma 7.1 yields the convergence $u^{n} \rightarrow u$, both in $L^{2}\left(0, T ; H_{\eta}^{\alpha / 2}\left(\mathbb{R}^{d}\right)\right)$ and in $C\left(0, T ; L_{\eta}^{2}\left(\mathbb{R}^{d}\right)\right)$, to the weak solution $u \in W^{1}\left(0, T ; H_{\eta}^{\alpha / 2}\left(\mathbb{R}^{d}\right), L_{\eta}^{2}\left(\mathbb{R}^{d}\right)\right)$ of

$$
\dot{u}+\mathcal{A}_{t} u+\kappa_{t} u=f, \quad u(0)=g .
$$

Lemma 8.1 shows that (8.5) holds pointwise and that $u^{n}$ is regular enough to apply Itô's formula. We denote by $\left(b_{t}, \sigma_{t}, F_{t} ; h\right)_{t \in[0, T]}$ the characteristics of $L$ and set $w^{n}(t, x):=u^{n}(T-t, x)$. Then Itô's formula for semimartingales entails

$$
\begin{aligned}
& w^{n}\left(T, L_{T}\right) \mathrm{e}^{-\int_{0}^{T} \kappa_{\lambda}^{n}\left(L_{\lambda}\right) \mathrm{d} \lambda}-w^{n}\left(s, L_{S}\right) \mathrm{e}^{-\int_{0}^{s} \kappa_{\lambda}^{n}\left(L_{\lambda}\right) \mathrm{d} \lambda} \\
& =\int_{s}^{T}\left(\dot{w^{n}}-\mathcal{A}_{\tau} w^{n}-\kappa w^{n}\right)\left(\tau, L_{\tau}\right) \mathrm{e}^{-\int_{0}^{\tau} \kappa_{\lambda}^{n}\left(L_{\lambda}\right) \mathrm{d} \lambda} \mathrm{d} \tau \\
& \quad+\int_{s}^{T}\left(\sigma_{\tau}^{1 / 2} \nabla w^{n}\left(\tau, L_{\tau}\right)\right) \mathrm{e}^{-\int_{0}^{\tau} \kappa_{\lambda}^{n}\left(L_{\lambda}\right) \mathrm{d} \lambda} \mathrm{d} W_{\tau} \\
& \quad+\left(\mathrm{e}^{-\int_{0}^{\cdot} \kappa_{\lambda}^{n}\left(L_{\lambda}\right) \mathrm{d} \lambda}\left(w^{n}\left(\cdot, L_{-}+x\right)-w^{n}\left(\cdot, L_{-}\right)\right) \mathbb{1}_{(s, \infty)}(\cdot)\right) *(\mu-v)_{T} .
\end{aligned}
$$

Thanks to our assumptions on $g^{n}, f^{n}$ and $\kappa^{n}$, we may decompose $u^{n}$ into three summands as in (8.3). Then, applying part (ii) of Lemma 8.1, it is elementary to conclude that $w^{n}$ and $\nabla w^{n}$ belong to $L^{2}\left(\mathbb{R}^{d}\right)$. Hence, the integrals with respect to $W$ and $\mu-v$ are martingales; compare [29, Theorem II.1.33 a)].

We insert $\dot{w}^{n}-\mathcal{A}_{\tau} w^{n}-\kappa^{n} w^{n}=\bar{f}^{n}$ with $\bar{f}(t, x):=f^{n}(T-t, x)$ in (8.6), subsequently multiply the equation with the term $\mathrm{e}_{0}^{s} \kappa_{\lambda}^{n}\left(L_{\lambda}\right) \mathrm{d} \lambda$ and finally take the conditional expectation. This gives for $0 \leq s \leq T$

$$
\begin{aligned}
& E\left[w^{n}\left(T, L_{T}\right) \mathrm{e}^{-\int_{s}^{T} \kappa_{\lambda}^{n}\left(L_{\lambda}\right) \mathrm{d} \lambda} \mid \mathcal{F}_{S}\right]-w^{n}\left(s, L_{s}\right) \\
& =E\left[\int_{s}^{T} \bar{f}^{n}\left(\tau, L_{\tau}\right) \mathrm{e}^{-\int_{s}^{\tau} \kappa_{\lambda}^{n}\left(L_{\lambda}\right) \mathrm{d} \lambda} \mathrm{d} \tau \mid \mathcal{F}_{S}\right] .
\end{aligned}
$$


Let without loss of generality $0<s \leq T$. We now derive the desired stochastic representation by letting $n \rightarrow \infty$ for each term in (8.7). Denote $w(t, x):=u(T-t, x)$. From the convergence $w^{n}(s, \cdot) \rightarrow w(s, \cdot)$ in $L_{\eta}^{2}\left(\mathbb{R}^{d}\right)$ and part (i) of Lemma 8.2 for $s>0$, we get the convergence

$$
w^{n}\left(s, L_{S}\right) \longrightarrow w\left(s, L_{s}\right) \quad \text { in } L^{1}(P) \text { and a.s. for a subsequence. }
$$

The pointwise convergence $\kappa^{n} \rightarrow \kappa$ and the uniform boundedness together with dominated convergence imply both $\int_{a}^{b} \kappa_{\lambda}^{n}\left(L_{\lambda}\right) \mathrm{d} \lambda \rightarrow \int_{a}^{b} \kappa_{\lambda}\left(L_{\lambda}\right) \mathrm{d} \lambda$ and uniform boundedness of the sequence for $0 \leq a \leq b \leq T$. Together with $w^{n}\left(s, L_{s}\right) \rightarrow w\left(s, L_{s}\right)$ in $L^{1}(P)$, the convergence

$$
E\left[\left|w^{n}\left(t, L_{t}\right) \mathrm{e}^{-\int_{s}^{T} \kappa_{\lambda}^{n}\left(L_{\lambda}\right) \mathrm{d} \lambda}-w\left(t, L_{t}\right) \mathrm{e}^{-\int_{s}^{T} \kappa_{\lambda}\left(L_{\lambda}\right) \mathrm{d} \lambda}\right| \mid \mathcal{F}_{S}\right] \longrightarrow 0
$$

as $n \rightarrow \infty$ then follows from the triangle inequality.

Next, denote $\bar{f}(t, x):=f(T-t, x)$. Since $f^{n} \rightarrow f$ in $L^{2}\left(t, T ; H_{\eta}^{\ell}\left(\mathbb{R}^{d}\right)\right)$, part (ii) of Lemma 8.2 guarantees the existence of a constant $c_{2}>0$ for $\ell>(d-\alpha) / 2$ such that

$$
E\left[\int_{s}^{T}\left|\left(\bar{f}^{n}-\bar{f}\right)\left(h, L_{h}\right)\right| \mathrm{d} h \mid \mathcal{F}_{S}\right] \leq c_{1}\left\|f^{n}-f\right\|_{L^{2}\left(t, T ; H_{\eta}^{\ell}\left(\mathbb{R}^{d}\right)\right)} \longrightarrow 0 .
$$

Now the triangle inequality yields the convergence of the second line in (8.7) and therefore (1.2) in part (ii) of Theorem 3.4 under the additional assumption that the mapping $t \mapsto A_{t}(\xi-i \eta)$ is continuous for every $\xi \in \mathbb{R}^{d}$. Finally, thanks to the tower rule of conditional expectations, this claim follows by induction over the continuity periods also under the more general assumption that $t \mapsto A_{t}(\xi-i \eta)$ is piecewise continuous for every $\xi \in \mathbb{R}^{d}$.

For every $t \in(0, T],(1.2)$ in particular shows that the conditional expectation on the left side is $\sigma\left(L_{t}\right)$-measurable. Hence it can be taken with respect to $L_{t}$. By Remark 4.1, $L_{t}$ has a (smooth) Lebesgue density; thus (3.8) follows.

Finally, defining the process $\widetilde{L}$ by its symbol $\widetilde{A}_{t}:=A_{0} \mathbb{1}_{\{t \leq \epsilon\}}+A_{t-\epsilon} \mathbb{1}_{\{t \geq \epsilon\}}$ and applying the result for the process $\widetilde{L}$ on the time interval $[0, T+\epsilon]$ yields the desired assertion for $t=0$.

Acknowledgements The roots of the present paper go back to the author's dissertation [17], which was financially supported by the DFG through project EB66/11-1. The author expresses her gratitude to Ernst Eberlein for his valuable support. The author also gratefully acknowledges Christoph Schwab and his working group for letting her use their wavelet-Galerkin implementation. She furthermore thanks Carsten Eilks, Paul Harrenstein, Alexandru Hening, Claudia Klüppelberg for valuable discussions and comments and the editors and the anonymous referees for their suggestions to improve the manuscript.

Open Access This article is distributed under the terms of the Creative Commons Attribution 4.0 International License (http://creativecommons.org/licenses/by/4.0/), which permits unrestricted use, distribution, and reproduction in any medium, provided you give appropriate credit to the original author(s) and the source, provide a link to the Creative Commons license, and indicate if changes were made. 


\section{Appendix A: Adjoint operator}

For a Lévy process $L$ with characteristics $(b, \sigma, F ; h)$, we denote by $\mathcal{A}^{(b, \sigma, F)}$ and $A^{(b, \sigma, F)}$ its Kolmogorov operator and its symbol, respectively. As the following assertions straightforwardly extend to the time-inhomogeneous case, we here present the time-homogeneous case only.

Lemma A.1 For $\eta \in \mathbb{R}^{d}$, let $L$ be a Lévy process with characteristics $(b, \sigma, F ; h)$ that satisfies the exponential moment condition (3.5) and denote by $\mathcal{A}$ its Kolmogorov operator with symbol A. Then

$$
A^{\eta}(\xi):=A(\xi+i \eta)=A^{\left(b^{\eta}, \sigma, F^{\eta}\right)}(\xi)+A(i \eta) \quad \text { for all } \xi \in \mathbb{R}^{d}
$$

with

$$
\begin{aligned}
b^{\eta} & =b+\sigma \eta+\int_{\mathbb{R}^{d}}\left(\mathrm{e}^{\langle\eta, y\rangle}-1\right) h(y) F(\mathrm{~d} y), \\
F^{\eta}(\mathrm{d} y) & =\mathrm{e}^{\langle\eta, y\rangle} F(\mathrm{~d} y) .
\end{aligned}
$$

In particular, $A^{\eta}$ is the symbol of a Lévy process with killing rate A(i $)$. Moreover, its Kolmogorov operator $\mathcal{A}^{\eta}$ satisfies

$$
\mathcal{A}^{\eta} \varphi=\mathrm{e}^{-\langle\eta, \cdot\rangle} \mathcal{A}\left(\mathrm{e}^{\langle\eta, \cdot\rangle} \varphi\right)=\mathcal{A}^{\left(b^{\eta}, \sigma, F^{\eta}\right)} \varphi+A(i \eta) \varphi \quad \text { for all } \varphi \in C_{0}^{\infty}\left(\mathbb{R}^{d}\right) .
$$

Proof It is elementary to verify the assertion for the symbol. This can then nicely be used to verify the assertion for the operator as follows. Let $\varphi \in C_{0}^{\infty}\left(\mathbb{R}^{d}\right)$; then $\mathcal{F}\left(\mathrm{e}^{\langle\eta, \cdot\rangle} \varphi\right)(\xi)=\mathcal{F}(\varphi)(\xi-i \eta)$ and

$$
\begin{aligned}
\mathcal{A}\left(\mathrm{e}^{\langle\eta, \cdot\rangle} \varphi\right)(x) & =\mathcal{F}^{-1}\left(A \mathcal{F}\left(\mathrm{e}^{\langle\eta, \cdot\rangle} \varphi\right)\right) \\
& =\frac{1}{(2 \pi)^{d}} \int_{\mathbb{R}^{d}} \mathrm{e}^{-i\langle\xi, x\rangle} A(\xi) \mathcal{F}(\varphi)(\xi-i \eta) \mathrm{d} \xi \\
& =\frac{\mathrm{e}^{\langle\eta, x\rangle}}{(2 \pi)^{d}} \int_{\mathbb{R}^{d}} \mathrm{e}^{-i\langle\xi, x\rangle} A(\xi+i \eta) \mathcal{F}(\varphi)(\xi) \mathrm{d} \xi
\end{aligned}
$$

which concludes the proof.

For all $\varphi \in C_{0}^{\infty}\left(\mathbb{R}^{d}\right)$, let

$$
\mathcal{F}_{\eta}(\varphi):=\mathrm{e}^{-\langle\eta, \cdot\rangle} \mathcal{F}\left(\varphi \mathrm{e}^{\langle\eta, \cdot\rangle}\right) \quad \text { and } \quad \mathcal{F}_{\eta}^{-1}(\varphi):=\mathrm{e}^{-\langle\eta, \cdot\rangle} \mathcal{F}^{-1}\left(\varphi \mathrm{e}^{\langle\eta, \cdot\rangle}\right) .
$$

Theorem 4.1 in [14] shows that for a pseudo-differential operator $\mathcal{A}$ whose symbol $A$ has a continuous extension to $U_{-\eta}$ that is analytic in the interior of $U_{-\eta}$ and satisfies the continuity condition (A2), we have

$$
\mathcal{A} \varphi=\mathcal{F}^{-1}(\mathcal{A} \varphi)=\mathcal{F}_{\eta}^{-1}\left(A^{-\eta} \mathcal{F}_{\eta}(\varphi)\right) \text { for all } \varphi \in C_{0}^{\infty}\left(\mathbb{R}^{d}\right) .
$$


Parseval's equality yields for all $\varphi, \psi \in C_{0}^{\infty}\left(\mathbb{R}^{d}\right)$ that

$$
a(\varphi, \psi)=\langle\mathcal{A} \varphi, \psi\rangle_{L_{\eta}^{2}}=\frac{1}{(2 \pi)^{d}}\left\langle A^{-\eta} \mathcal{F}_{\eta}(\varphi), \mathcal{F}_{\eta}(\psi)\right\rangle_{L_{\eta}^{2}}
$$

For the pseudo-differential operator $\mathcal{A}$ and its symbol $A$, we define their $L_{\eta}^{2}$-adjoints $\mathcal{A}^{\eta, *}$ and $A^{-\eta, *}$ such that for all $\varphi, \psi \in C_{0}^{\infty}\left(\mathbb{R}^{d}\right)$,

$$
\begin{aligned}
\langle\mathcal{A}, \psi\rangle_{L_{\eta}^{2}} & =\left\langle\varphi, \mathcal{A}^{\eta, *} \psi\right\rangle_{L_{\eta}^{2}}, \\
\left\langle A^{-\eta} \mathcal{F}_{\eta}(\varphi), \mathcal{F}_{\eta}(\psi)\right\rangle_{L_{\eta}^{2}} & =\left\langle\mathcal{F}_{\eta}(\varphi), A^{-\eta, *} \mathcal{F}_{\eta}(\psi)\right\rangle_{L_{\eta}^{2}}
\end{aligned}
$$

Lemma A.2 For $\eta \in \mathbb{R}^{d}$, let $L$ be a Lévy process with characteristics $(b, \sigma, F ; h)$ that satisfies the exponential moment condition (3.5) and denote by $\mathcal{A}$ its Kolmogorov operator with symbol $A$. Using the notations $F_{\mathrm{sym}}^{-\eta}(B)=\left(F^{-\eta}(B)+F^{-\eta}(-B)\right) / 2$ and $F_{\text {asym }}^{-\eta}(B)=F^{-\eta}(B)-F_{\text {sym }}^{-\eta}(B)$, set $F^{-\eta, *}(B)=F_{\text {sym }}^{-\eta}(B)-F_{\text {asym }}^{-\eta}(B)$ for Borel sets $B \neq\{0\}$, and let $b^{-\eta, *}=-b^{-\eta}$. Then

$$
\begin{aligned}
A^{-\eta, *} & =\overline{A^{-\eta}}=A^{\left(b^{-\eta, *}, \sigma, F^{-\eta, *}\right)}+A(-i \eta), \\
\mathcal{A}^{-\eta, *} \varphi & =\mathrm{e}^{-\langle\eta, \cdot\rangle} \mathcal{A}\left(\mathrm{e}^{\langle\eta, \cdot\rangle} \varphi\right)=\mathcal{A}^{\left(b^{-\eta, *}, \sigma, F^{-\eta, *}\right)} \varphi+A(-i \eta) \varphi .
\end{aligned}
$$

Moreover, $F^{-\eta, *}$ is a Lévy measure.

Proof For every $\varphi \in C_{0}^{\infty}\left(\mathbb{R}^{d}\right)$, we have

$$
\begin{aligned}
\left\langle A^{-\eta} \mathcal{F}_{\eta}(\varphi), \mathcal{F}_{\eta}(\psi)\right\rangle_{L_{\eta}^{2}} & =\left\langle A^{-\eta} \mathcal{F}\left(\mathrm{e}^{\langle\eta, \cdot\rangle} \varphi\right), \mathcal{F}\left(\mathrm{e}^{\langle\eta, \cdot\rangle} \varphi\right)\right\rangle_{L^{2}} \\
& =\left\langle\mathcal{F}\left(\mathrm{e}^{\langle\eta, \cdot\rangle} \varphi\right), \overline{A^{-\eta}} \mathcal{F}\left(\mathrm{e}^{\langle\eta, \cdot\rangle} \varphi\right)\right\rangle_{L^{2}} .
\end{aligned}
$$

Since $A(z) \in \mathbb{R}$ for $z \in \mathbb{C}^{d}$ with $\Re(z)=0$, by Lemma A.1 we obtain

$$
\overline{A^{-\eta}}=\overline{A^{\left(b^{-\eta}, \sigma, F^{-\eta}\right)}}+A(-i \eta) .
$$

As $A^{\left(b^{-\eta}, \sigma, F^{-\eta}\right)}$ is the symbol of a Lévy process, we have

$$
\overline{A^{\left(b^{-\eta}, \sigma, F^{-\eta}\right)}(\xi)}=A^{\left(b^{-\eta}, \sigma, F^{-\eta}\right)}(-\xi) \quad \text { for all } \xi \in \mathbb{R}^{d}
$$

whence the assertion of the lemma follows directly.

\section{Appendix B: Proof of Theorem 3.3}

Theorem B.1 For $\eta \in \mathbb{R}^{d}$ and $\alpha \in(0,1]$, let the symbol $A=\left(A_{t}\right)_{t \in[0, T]}$ of the pseudo-differential operator $\mathcal{A}=\left(\mathcal{A}_{t}\right)_{t \in[0, T]}$ be such that for each $t \in[0, T], A_{t}$ has 
a continuous extension on $U_{-\eta}$ that is analytic in the interior $U_{-\eta}$ and such that there exist constants $C(t), m(t)>0$ with

$$
\left|A_{t}(z)\right| \leq C(t)(1+|z|)^{m(t)} \quad \text { for all } z \in U_{-\eta} .
$$

Then the following assertions are equivalent:

(i) The operator $\mathcal{A}$ is parabolic with respect to $\left(H_{\eta^{\prime}}^{\alpha}\left(\mathbb{R}^{d}\right), L_{\eta^{\prime}}^{2}\left(\mathbb{R}^{d}\right)\right)_{\eta^{\prime} \in R_{\eta}}$ uniformly in $[0, T] \times R_{\eta}$.

(ii) The symbol A has Sobolev index $2 \alpha$ uniformly in $[0, T] \times R_{\eta}$.

The proof of the theorem is a straightforward generalization of the proof of [18, Theorem 3.1], where the assertion is proved for symbols that are constant in time and for spaces without weights, that is, if $\eta=0$. In order to provide a self-contained presentation, we give a detailed proof.

Proof of Theorem B.1 By the assumption on the analyticity of $A$ and (B.1), we obtain from [14, Theorem 4.1] that for all $t \in[0, T], \eta^{\prime} \in R_{\eta}$ and $\varphi, \psi \in C_{0}^{\infty}\left(\mathbb{R}^{d}\right)$,

$$
\begin{aligned}
a_{t}(\varphi, \psi) & =\frac{1}{(2 \pi)^{d}}\left\langle A_{t} \mathcal{F}(\varphi), \mathcal{F}(\psi)\right\rangle_{L^{2}}=\frac{1}{(2 \pi)^{d}}\left\langle A_{t}\left(\cdot-i \eta^{\prime}\right) \mathcal{F}_{\eta^{\prime}}(\varphi), \mathcal{F}_{\eta^{\prime}}(\psi)\right\rangle_{L_{\eta^{\prime}}^{2}} \\
& =\frac{1}{(2 \pi)^{d}} \int_{\mathbb{R}^{d}} \overline{A_{t}\left(\xi-i \eta^{\prime}\right) \mathcal{F}(\varphi)\left(\xi-i \eta^{\prime}\right)} \mathcal{F}(\psi)\left(\xi-i \eta^{\prime}\right) \mathrm{d} \xi .
\end{aligned}
$$

This equality yields that (3.6) implies (3.1). Together with the following elementary inequalities, it also yields that (3.7) implies (3.2): For $C_{1}>0, C_{2} \geq 0,0 \leq \beta<\alpha$ and $0<C_{3}<C_{1}$, there exists a constant $C_{4}>0$ such that $C_{1} x^{\alpha}-C_{2} x^{\beta} \geq C_{3} x^{\alpha}-C_{4}$ for all $x \geq 0$ and

$$
C_{2}|\xi|^{2 \alpha}-C_{3}\left(1+|\xi|^{2}\right)^{\beta} \geq C_{2}|\xi|^{2 \alpha}-C_{3}^{\prime}\left(1+|\xi|^{2 \beta}\right) \geq c_{2}(1+|\xi|)^{2 \alpha}-c_{3}
$$

with a strictly positive constant $c_{2}$ and $C_{3}^{\prime}, c_{3} \geq 0$. Moreover, the piecewise continuity of $t \mapsto a_{t}(u, v)$ for every $u, v \in H_{\eta}^{\alpha}\left(\mathbb{R}^{d}\right)$ follows from the piecewise continuity of $t \mapsto A_{t}(z)$ for every $z \in U_{-\eta}$ and dominated convergence, which applies by (3.6).

In order to derive the implication from (i) to (ii), we first show the following. Let $\gamma: \mathbb{R}^{d} \rightarrow \mathbb{R}$ be a continuous function. If we have

$$
\int_{\mathbb{R}^{d}} \gamma(\xi)\left|\mathcal{F}_{\eta^{\prime}}(u)(\xi)\right|^{2} \mathrm{e}^{-2\left\langle\eta^{\prime}, \xi\right\rangle} \mathrm{d} \xi \geq 0
$$

for all $u \in H_{\eta^{\prime}}^{\alpha}\left(\mathbb{R}^{d}\right)$ for which $\mathcal{F}_{\eta^{\prime}}(u)$ is compactly supported, then $\gamma(\xi) \geq 0$ for all $\xi \in \mathbb{R}^{d}$. To prove this claim, we follow closely the derivation of the fundamental lemma of variational calculus. Let us for a moment assume that $\gamma(\xi)<0$ for some $\xi \in \mathbb{R}^{d}$. Due to continuity, $\gamma$ is negative on a nonempty open subset of $U \subset \mathbb{R}^{d}$. We now choose a function $u \in H_{\eta^{\prime}}^{\alpha}\left(\mathbb{R}^{d}\right)$ such that its Fourier transform $\mathcal{F}_{\eta^{\prime}}(u)$ is smooth, nonconstant and compactly supported in $U$. For this choice of $u$, however, the integral in (B.3) would be negative, leading to a contradiction. This shows that $\gamma \geq 0$. 
To get (3.6), we observe that (3.1) implies (B.3) for the continuous mappings $\xi \mapsto C(1+|\xi|)^{2 \alpha} \pm \Re\left(A_{t}\left(\xi-i \eta^{\prime}\right)\right)$ and $\xi \mapsto C(1+|\xi|)^{2 \alpha} \pm \Im\left(A_{t}\left(\xi-i \eta^{\prime}\right)\right)$ for all $t \in[0, T]$ and $\eta^{\prime} \in R_{\eta}$. Thus (3.6) follows. Similarly, using once again (B.2), we obtain that (3.2) implies (3.7).

Finally, we observe that $\lim _{s \rightarrow t} a_{s}(u, u)=a_{t}(u, u)$ implies that

$$
\lim _{s \rightarrow t} \int_{\mathbb{R}^{d}} A_{s}\left(\xi-i \eta^{\prime}\right)\left|\mathcal{F}_{\eta^{\prime}}(u)(\xi)\right|^{2} \mathrm{~d} \xi=\int_{\mathbb{R}^{d}} A_{t}\left(\xi-i \eta^{\prime}\right)\left|\mathcal{F}_{\eta^{\prime}}(u)(\xi)\right|^{2} \mathrm{~d} \xi,
$$

while on the other hand dominated convergence shows that

$$
\lim _{s \rightarrow t} \int_{\mathbb{R}^{d}} A_{s}\left(\xi-i \eta^{\prime}\right)\left|\mathcal{F}_{\eta^{\prime}}(u)(\xi)\right|^{2} \mathrm{~d} \xi=\int_{\mathbb{R}^{d}} \lim _{s \rightarrow t} A_{s}\left(\xi-i \eta^{\prime}\right)\left|\mathcal{F}_{\eta^{\prime}}(u)(\xi)\right|^{2} \mathrm{~d} \xi .
$$

Now, an application of (B.3) yields $\lim _{s \rightarrow t} A_{s}\left(\xi-i \eta^{\prime}\right)=A_{t}\left(\xi-i \eta^{\prime}\right)$ for all $\xi \in \mathbb{R}^{d}$ and $\eta^{\prime} \in R_{\eta}$. Therefore, piecewise continuity of the bilinear form entails piecewise continuity of the symbol.

Theorem 3.3 now follows as a Corollary from Theorem B.1:

Proof of Theorem 3.3 According to Lemma 2.1 (c) in [14], for every $0 \leq t \leq T$, the map $z \mapsto A_{t}(z)$ has a continuous extension to the domain $\overline{U_{-\eta}}$. Moreover, Theorem 25.17 in [42], together with Lemma A.1, shows that (B.1) is satisfied for each $t \in[0, T]$ with $m(t)=2$ and some constant $C(t)>0$. Theorem 3.3 is now applicable and yields the corollary.

\section{References}

1. Aït-Sahalia, Y., Jacod, J.: High-Frequency Financial Econometrics, vol. 1, 1st edn. Princeton University Press, Princeton (2014)

2. Albrecher, H., Lautscham, V.: From ruin to bankruptcy for compound Poisson surplus processes. ASTIN Bull. 43, 213-243 (2013)

3. Albrecher, H., Gerber, H., Shiu, E.: The optimal dividend barrier in the Gamma-Omega model. Eur. Actuar. J. 1(1), 43-56 (2011)

4. Asmussen, S., Rosiński, J.: Approximations of small jumps of Lévy processes with a view towards simulation. J. Appl. Probab. 38, 482-493 (2001)

5. Bensoussan, A., Lions, J.L.: Contrôle Impulsionnel et Inéquations Quasi Variationnelles. GauthierVillars, Paris (1982)

6. Black, F., Scholes, M.: The pricing of options and corporate liabilities. J. Polit. Econ. 81, 637-654 (1973)

7. Böttcher, B., Schilling, R., Wang, J.: Lévy Matters III. Lévy-Type Processes: Construction, Approximation and Sample Path Properties. Lecture Notes in Mathematics. Springer, Berlin (2013). 2099

8. Boyarchenko, S.I., Levendorskiı̌, S.Z.: Barrier options and touch-and-out options under regular Lévy processes of exponential type. Ann. Appl. Probab. 12, 1261-1298 (2002)

9. Cont, R., Tankov, P.: Financial Modelling with Jump Processes. Financial Mathematics. Chapman \& Hall /CRC Press, London/Boca Raton (2004)

10. Cont, R., Voltchkova, E.: A finite difference scheme for option pricing in jump diffusion and exponential Lévy models. SIAM J. Numer. Anal. 43, 1596-1626 (2005)

11. Cont, R., Voltchkova, E.: Integro-differential equations for option prices in exponential Lévy models. Finance Stoch. 9, 299-325 (2005) 
12. Cont, R., Lantos, N., Pironneau, O.: A reduced basis for option pricing. SIAM J. Financ. Math. 2, 287-316 (2011)

13. Eberlein, E.: Application of generalized hyperbolic Lévy motions to finance. In: Barndorff-Nielsen, O.E., et al. (eds.) Lévy Processes, pp. 319-336. Birkhäuser, Basel (2001)

14. Eberlein, E., Glau, K.: Variational solutions of the pricing PIDEs for European options in Lévy models. Appl. Math. Finance 21, 417-450 (2014)

15. Eberlein, E., Kluge, W.: Exact pricing formulae for caps and swaptions in a Lévy term structure model. J. Comput. Finance 9(2), 99-125 (2006)

16. Eberlein, E., Raible, S.: Term structure models driven by general Lévy processes. Math. Finance $\mathbf{9}$, 31-53 (1999)

17. Glau, K.: Feynman-Kac-Darstellung zur Optionspreisbewertung in Lévy-Modellen. PhD thesis, Universität Freiburg (2010). Available online at https://www.freidok.uni-freiburg.de/data/7724

18. Glau, K.: Classification of Lévy processes with parabolic Kolmogorov backward equations. SIAM J. Theory Probab. Appl. (2016, forthcoming). Available online at http://arxiv.org/abs/1203.0866

19. Haasdonk, B., Salomon, J., Wohlmuth, B.: A reduced basis method for parametrized variational inequalities. SIAM J. Numer. Anal. 50, 2656-2676 (2012)

20. Burkovska, O., Haasdonk, B., Salomon, J., Wohlmuth, B.: Reduced basis methods for pricing options with the Black-Scholes and Heston models. SIAM J. Financ. Math. 6, 685-712 (2015)

21. Hammerstein, E.A.: Generalized Hyperbolic Distributions: Theory and Applications to CDO Pricing. PhD thesis, Universität Freiburg (2010). Available online at https://www.freidok.uni-freiburg.de/ data/7974

22. Hilber, N., Reich, N., Winter, C., Schwab, C.: Numerical methods for Lévy processes. Finance Stoch. 13, 471-500 (2009)

23. Hilber, N., Reichmann, O., Schwab, C., Winter, C.: Computational Methods for Quantitative Finance. Springer, Berlin (2013)

24. Hoh, W.: The martingale problem for a class of pseudo differential operators. Math. Ann. 300, 121147 (1994)

25. Itkin, A.: Efficient solution of backward jump-diffusion PIDEs with splitting and matrix exponentials. J. Comput. Finance 19(3), 29-70 (2016)

26. Jacob, N.: Pseudo Differential Operators and Markov Processes, vol I. Fourier Analysis and Semigroups. Imperial College Press, London (2001)

27. Jacob, N.: Pseudo Differential Operators and Markov Processes, vol II. Generators and Their Potential Theory. Imperial College Press, London (2002)

28. Jacob, N.: Pseudo Differential Operators and Markov Processes, vol III. Markov Processes and Applications. Imperial College Press, London (2005)

29. Jacod, J., Shiryaev, A.N.: Limit Theorems for Stochastic Processes, 2nd edn. Springer, Berlin (2003)

30. Jeanblanc, M., Le Cam, Y.: Reduced form modelling for credit risk. Université d'Evry Val d'Essonne (2007, preprint). Available online at http://www.defaultrisk.com/pp_model135.htm

31. Kac, M.: On distributions of certain Wiener functionals. Trans. Am. Math. Soc. 65, 1-13 (1949)

32. Landriault, D., Renaud, J.R., Zhou, X.: Occupation times of spectrally negative Lévy processes. In: Stochastic Processes and Their Applications, vol. 121, pp. 2629-2641 (2011)

33. Matache, A.M., von Petersdorff, T., Schwab, C.: Fast deterministic pricing of options on Lévy driven assets. Math. Model. Numer. Anal. 38, 37-71 (2004)

34. Matache, A.M., Schwab, C., Wihler, T.P.: Fast numerical solution of parabolic integrodifferential equations with applications in finance. SIAM J. Sci. Comput. 27, 369-393 (2005)

35. Nezza, E.D., Palatucci, G., Valdinoci, E.: Hitchhiker's guide to the fractional Sobolev spaces. Bull. Sci. Math. 136, 521-573 (2012)

36. von Petersdorff, T., Schwab, C.: Wavelet discretizations of parabolic integrodifferential equations. SIAM J. Numer. Anal. 41, 159-180 (2003)

37. von Petersdorff, T., Schwab, C.: Numerical solution of parabolic equations in high dimensions. Math. Model. Numer. Anal. 38, 93-127 (2004)

38. Pironneau, O.: Reduced basis for vanilla and basket options. Risk Decis. Anal. 2(4), 185-194 (2011)

39. Poirot, J., Tankov, P.: Monte Carlo option pricing for tempered stable (CGMY) processes. Asia-Pac. Financ. Mark. 13, 327-344 (2006)

40. Sachs, E.W., Schu, M.: A priori error estimates for reduced order models in finance. Math. Model. Numer. Anal. 47, 449-469 (2013)

41. Salmi, S., Toivanen, J., von Sydow, L.: An IMEX-scheme for pricing options under stochastic volatility models with jumps. SIAM J. Sci. Comput. 36, B817-B834 (2014) 
42. Sato, K.I.: Lévy Processes and Infinitely Divisible Distributions. Cambridge University Press, Cambridge (1999)

43. Schoutens, W.: Lévy Processes in Finance: Pricing Financial Derivatives. Wiley, New York (2003)

44. Wloka, J.: Partial Differential Equations. Cambridge University Press, Cambridge (1987)

45. Zeidler, E.: Nonlinear Functional Analysis and Its Applications, vol II/A. Linear Monotone Operators. Springer, Berlin (1990) 